\title{
A Note on the Minimum Size of a Point Set Containing Three Nonintersecting Empty Convex Polygons
}

\author{
Qing Yang ${ }^{1}$, Zengtai You ${ }^{2}$ and Xinshang You ${ }^{3, *}$ \\ 1 Faculty of Accounting, Shanxi University of Finance and Economics, Taiyuan 030006, China; \\ yangqing@tju.edu.cn \\ 2 College of Computer Science and Engineering, Dalian Minzu University, Dalian 116600, China; \\ zengtaiyou@163.com \\ 3 College of Economics and Management, Shandong University of Science and Technology, \\ Qingdao 266590, China \\ * Correspondence: youxinshang@tju.edu.cn
}

Received: 6 September 2018; Accepted: 26 September 2018; Published: 29 September 2018

\begin{abstract}
Let $P$ be a planar point set with no three points collinear, $k$ points of $P$ be a $k$-hole of $P$ if the $k$ points are the vertices of a convex polygon without points of $P$. This article proves 13 is the smallest integer such that any planar points set containing at least 13 points with no three points collinear, contains a 3-hole, a 4-hole and a 5-hole which are pairwise disjoint.
\end{abstract}

Keywords: planar point set; convex polygon; disjoint holes

\section{Introduction}

In this paper, we deal with the finite planar point set $P$ in general position, that is to say, no three points in $P$ are collinear. In 1935, Erdős and Szekeres [1], posed a famous combinational geometry question: Whether for every positive integer $m \geq 3$, there exists a smallest integer $E S(m)$, such that any set of $n$ points $(n \geq E S(m))$, contains a subset of $m$ points which are the vertices of a convex polygon. It is a long standing open problem to evaluate the exact value of $E S(m)$. Erdős and Szekeres [2] showed that $E S(m) \geq 2^{m-2}+1$, which is also conjectured to be sharp. We have known that $E S(4)=5$ and $E S(5)=9$. Then by using computer, Szekeres and Peters [3] proved that $E S(6)=17$. The value of $E S(m)$ for all $m>6$ is unknown.

For a planar point set $P$, let $k$ points of $P$ be a $k$-hole of $P$ if the $k$ points are the vertices of a convex polygon whose interior contains no points of $P$. Erdős posed another famous question in 1978. He asked whether for every positive integer $k$, there exists a smallest integer $H(k)$, such that any set of at least $H(k)$ points in the plane, contains a $k$-hole. It is obvious that $H(3)=3$. Esther Klein showed $H(4)=5$. Harborth [4] determined $H(5)=10$, and also gave the configuration of nine points with no empty convex pentagons. Horton [5] showed that it was possible to construct arbitrarily large set of points without a 7-hole, That is to say $H(k)$ does not exist for $k \geq 7$. The existence of $H(6)$ had been proved by Gerken [6] and Nicolás [7], independently. In [8], Urabe first studied the disjoint holes problems when hewas considering the question about partitioning of planar point sets. Let $C h(P)$ stand for the convex hull of a point set $P$. A family of holes $\left\{H_{i}\right\}_{i \in I}$ is called pairwise disjoint if $C h\left(H_{i}\right) \cap C h\left(H_{j}\right)=\varnothing, i \neq j ; i \in I, j \in I$. These holes are disjoint with each other. Determine the smallest integer $n\left(k_{1}, \ldots, k_{l}\right), k_{1} \leq k_{2} \leq \ldots \leq k_{l}$, such that any set of at least $n\left(k_{1}, \ldots, k_{l}\right)$ points of the plane, contains a $k_{i}$-hole for every $i, 1 \leq i \leq l$, where the holes are disjoint. From [9], we know $n(2,4)=6, n(3,3)=6$. Urabe [8] showed that $n(3,4)=7$, while Hosono and Urabe [10] showed that $n(4,4)=9$. In [11], Hosono and Urabe also gave $n(3,5)=10,12 \leq n(4,5) \leq 14$ and $16 \leq n(5,5) \leq 20$. The result $n(3,4)=7$ and $n(4,5) \leq 14$ were re-authentication by $\mathrm{Wu}$ and Ding [12]. Hosono and 
Urabe [9] proved $n(4,5) \leq 13 . n(4,5)=12$ by Bhattacharya and Das was published in [13], who also discussed the convex polygons and pseudo-triangles [14]. Hosono and Urabe also changed the lower bound on $H(5,5)$ to 17 [9], and Bhattacharya and Das showed the upper bound on $n(5,5)$ to 19 [15]. Recently, more detailed discussions about two holes are published in [16]. Hosono and Urabe in [9] showed $n(2,3,4)=9, n(2,3,5)=11, n(4,4,4)=16$. We showed $n(3,3,5)=12$ in [17]. We have proved that $n(3,3,5)=12$ [17], $n(4,4,5) \leq 16$ [18] and also discuss a disjoint holes problem in preference [19]. In this paper, we will continue discussing this problem and prove that $n(3,4,5)=13$.

\section{Definitions}

The vertices are on convex hull of the given points,from the remaining interior points. Let $V(P)$ denote a set of the vertices and $I(P)$ be a set of the interior points of $P .|P|$ stands for the number of points contained in $P$. Let $p_{1}, p_{2}, \ldots, p_{k}$ be $k$ points of $P$, we know that $p_{1}, p_{2}, \ldots, p_{k}$ be a $k$-hole $H$ when the $k$ points are the vertices of a convex polygon whose interior does not contain any point of $P$. And we simply say $H=\left(p_{1} p_{2} \ldots p_{k}\right)_{k}$. As in [9], let $l(a, b)$ be the line passing points $a$ and $b$. Determine the closed half-plane with $l(a, b)$, who contains $c$ or does not contain $c$ by $H(c ; a b)$ or $H(\bar{c} ; a b)$, respectively. $R$ is a region in the plane. An interior point of $R$ is an element of a given point set $P$ in its interior, and we say $R$ is empty when $R$ contains no interior points, and simply $R=\varnothing$. The interior region of the angular domain determined by the points $a, b$ and $c$ is a convex cone. It is denoted by $\gamma(a ; b, c) . a$ is the apex. $b$ and $c$ are on the boundary of the angular domain. If $\gamma(a ; b, c)$ is not empty, we define an interior point of $\gamma(a ; b, c)$ be attack point $\alpha(a ; b, c)$, such that $\gamma(a ; b, \alpha(a ; b, c))$ is empty, as shown in Figure 1.

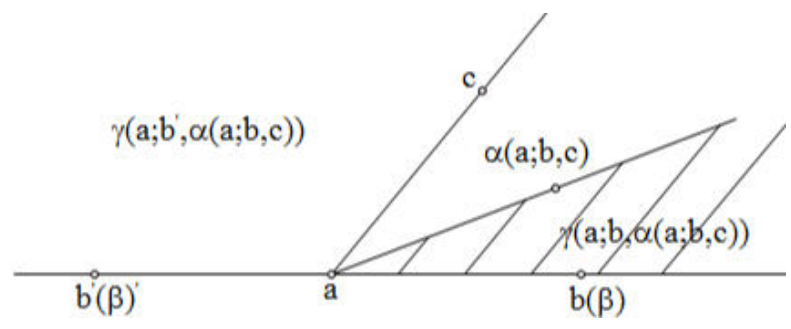

Figure 1. Figure of attack point.

For $\beta=b$ or $\beta=c$ of $\gamma(a ; b, c)$, let $\beta^{\prime}$ be a point such that $a$ is on the line segment $\overline{\beta \beta^{\prime}} . \gamma\left(a ; b^{\prime}, c\right)$ means that $a$ lies on the segment $\overline{b b^{\prime}}$. Let $v_{1}, v_{2}, v_{3}, v_{4} \in P$ and $\left(v_{1} v_{2} v_{3} v_{4}\right)_{4}$ be a 4-hole, as shown in Figure 2. We name $l\left(v_{3}, v_{4}\right)$ a separating line, denoted by $S L\left(v_{3}, v_{4}\right)$ or $S L_{4}$ for simple, when all of the remaining points of $P$ locate in $H\left(\overline{v_{1}} ; v_{3} v_{4}\right)$.

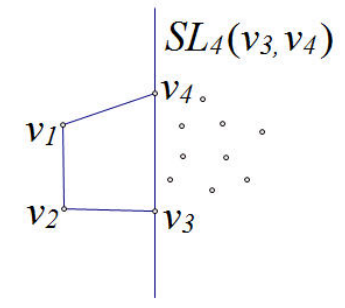

Figure 2. Figure of separating line.

We identify indices modulo $t$, when indexing a set of $t$ points. 


\section{Main Result and Proof}

Theorem 1. [9] For any planar point set with at least 13 points in general position, if there exists a separating line $S L_{4}$, which separates a 4-hole from all of the remaining points, we always can find a 3-hole, a 4-hole and a 5-hole which are pairwise disjoint.

From [20], we know that $13 \leq n(3,4,5) \leq 14$. In this note we will give the exact value of $n(3,4,5)$, that is the following theorem.

Theorem 2. $n(3,4,5)=13$, that is to say, 13 is the smallest integer such that any planar point set with at least 13 points in general position, we always can find a 3-hole, a 4-hole and a 5-hole which are pairwise disjoint.

Proof. Let $P$ be a 13 points set. $C H(P)=\left\{v_{1}, v_{2}, \ldots, v_{l}\right\}$. If we can find a 5-hole and a disjoint convex region with at least 7 points remained, we are done by $n(3,4)=7$ [8]. That is to say, if we find a straight line which separates a 5-hole from at least 7 points remained, the result is correct. We call such a line a cutting line through two points $u$ and $v$ in $P$, denoted by $L_{5}(u, v)$. If we can find a 4-hole and the vertices number of the remaining points is more than 4 , we are done by Theorem 1 , where the two parts are disjoint. That is to say, if we can find such a cutting line through two points $m$ and $n$ in $P$, denoted by $L_{4}(m, n)$, our conclusion is correct. Therefore, in the following proof, if we can find a cutting line $L_{5}(u, v)$ or $L_{4}(m, n)$, our conclusion must be true.

In the following, we will assume there does not exist a separating line $S L_{4}$. Then there must exist a point $p_{i}$, such that $\gamma\left(p_{i} ; v_{i}, v_{i-1}^{\prime}\right)$ and $\gamma\left(p_{i} ; v_{i-1}, v_{i}^{\prime}\right)$ are empty, as shown in Figure 3 . Considering the 13 points, it is easy to know the conclusion is obvious right when $|V(P)| \geq 7$. Next, we discuss the considerations that $3 \leq|V(P)| \leq 6$.

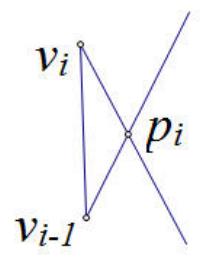

Figure 3. Figure of point determined by two separating lines.

Case $1|V(P)|=6$.

Let $v_{i} \in V(P)$ for $i=1,2, \ldots 6$. As shown in Figure 4 , we have the points $p_{i}$ for $i=1,2, \ldots 6$, such that the shaded region is empty and we have 1 point $p_{7}$ remained.

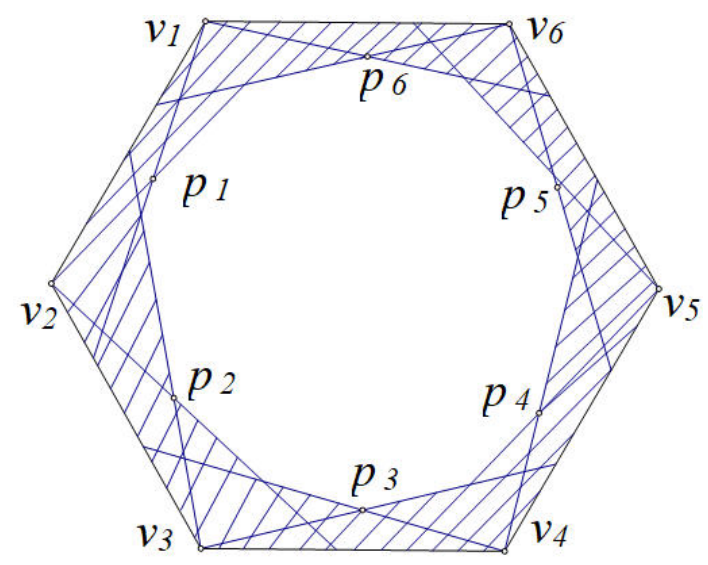

Figure 4. Figure of $|\mathrm{V}(\mathrm{P})|=6$ 
As the isomorphism of geometry from Figure 4, we only discuss one case. And the rest could be obtained in the same way.

Assume $\gamma\left(v_{1} ; p_{1}, v_{3}\right) \cap \gamma\left(v_{3} ; v_{1}, p_{2}\right)=\varnothing$. We have a cutting line $L_{5}\left(v_{1}, \alpha\left(v_{1} ; v_{3}, v_{6}\right)\right)$.

Assume $\gamma\left(v_{1} ; p_{1}, v_{3}\right) \cap \gamma\left(v_{3} ; v_{1}, p_{2}\right) \neq \varnothing$. We have a cutting line $L_{5}\left(v_{1}, p_{4}\right)$.

Case $2|V(P)|=5$.

Let $v_{i} \in V(P)$ for $i=\mathbf{1}, \mathbf{2}, \mathbf{3}, \mathbf{4}, \mathbf{5}$. We have 5 friend points $p_{i}$ for $i=\mathbf{1}, \mathbf{2}, \mathbf{3}, \mathbf{4}, \mathbf{5}$ as shown in Figure 5. Then we have 3 points $r_{1}, r_{2}, r_{3}$ remained.

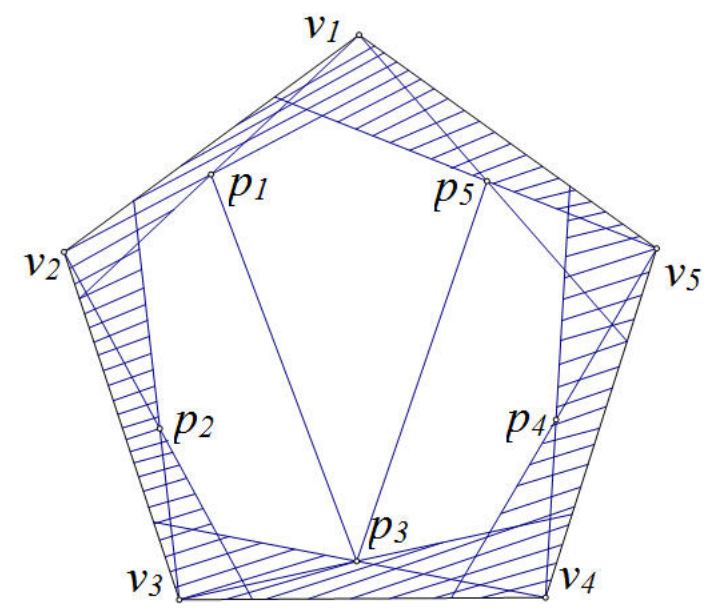

Figure 5. Figure of $|V(P)|=5$.

Assume $\gamma\left(p_{1} ; v_{1}^{\prime}, p_{3}\right) \cap \gamma\left(p_{2} ; v_{3}^{\prime}, v_{2}^{\prime}\right)=\varnothing$. We have a cutting line $L_{5}\left(p_{1}, \alpha\left(p_{1} ; p_{3}, v_{2}^{\prime}\right)\right)$.

Assume $\gamma\left(p_{3} ; v_{3}^{\prime}, p_{5}\right) \cap \gamma\left(p_{4} ; v_{4}^{\prime}, v_{5}^{\prime}\right)=\varnothing$. We have a cutting line $L_{5}\left(p_{3}, \alpha\left(p_{3} ; p_{5}, p_{1}\right)\right)$.

Assume $\gamma\left(p_{1} ; v_{1}^{\prime}, p_{3}\right) \cap \gamma\left(p_{2} ; v_{3}^{\prime}, v_{2}^{\prime}\right) \neq \varnothing$ and $\gamma\left(p_{3} ; v_{3}^{\prime}, p_{5}\right) \cap \gamma\left(p_{4} ; v_{4}^{\prime}, v_{5}^{\prime}\right) \neq \varnothing$. Suppose $\gamma\left(p_{1} ; v_{2}^{\prime}, p_{3}\right) \cap \gamma\left(p_{5} ; v_{5}^{\prime}, p_{3}\right)=\varnothing$. If $\gamma\left(p_{1} ; v_{1}^{\prime}, p_{3}\right) \cap \gamma\left(p_{2} ; v_{3}^{\prime}, v_{2}^{\prime}\right)$ has two of the remaining points say $r_{1}, r_{2}, r_{3} \in \gamma\left(p_{5} ; p_{3}, v_{5}\right)$, let $r_{1}=\alpha\left(p_{3} ; p_{1}, v_{4}^{\prime}\right):$ and if $r_{2} \in \gamma\left(r_{1} ; p_{2}, p_{3}^{\prime}\right) \neq \varnothing$, we have a cutting line $L_{5}\left(r_{1}, p_{3}\right)$; and if $r_{2} \in \gamma\left(r_{1} ; p_{1}^{\prime}, p_{3}\right)$, we have $\left(v_{2} v_{3} p_{2}\right)_{3},\left(p_{1} r_{1} r_{2} p_{3} v_{1}\right)_{5}$ and a 4-hole from the remaining points; and if $r_{2} \in \gamma\left(r_{1} ; p_{2}, p_{1}^{\prime}\right)$, we have a cutting line $L_{5}\left(p_{1}, r_{1}\right)$. If $\gamma\left(p_{5} ; p_{3}, v_{5}\right) \cap \gamma\left(v_{4} ; p_{3}, p_{4}\right)$ has two of the remaining points, symmetrically, the conclusion is also right. Suppose $\gamma\left(p_{1} ; v_{2}^{\prime}, p_{3}\right) \cap \gamma\left(p_{5} ; v_{5}^{\prime}, p_{3}\right) \neq \varnothing$. We may suppose $r_{1} \in \gamma\left(p_{1} ; v_{1}^{\prime}, p_{3}\right) \cap \gamma\left(p_{2} ; v_{3}^{\prime}, v_{2}^{\prime}\right)$, $r_{2} \in \gamma\left(p_{1} ; v_{2}^{\prime}, p_{3}\right) \cap \gamma\left(p_{5} ; v_{5}^{\prime}, p_{3}\right), r_{3} \in \gamma\left(p_{3} ; v_{4}, p_{5}\right) \cap \gamma\left(p_{4} ; v_{4}^{\prime}, v_{5}^{\prime}\right)$. If $\gamma\left(r_{2} ; p_{1}, p_{3}^{\prime}\right) \neq \varnothing$, we have $\left(v_{2} v_{3} p_{2}\right)_{3},\left(p_{1} r_{1} p_{3} r_{2} v_{1}\right)_{5}$ and a 4-hole from the remaining points. If $\gamma\left(r_{2} ; p_{3}, p_{1}^{\prime}\right) \neq \varnothing$, we have $\left(v_{2} v_{3} p_{2}\right)_{3},\left(r_{2} p_{1} r_{1} p_{3} \alpha\left(r_{2} ; p_{3}, p_{1}^{\prime}\right)\right)_{5}$ and a 4 -hole from the remaining points. If $\gamma\left(r_{2} ; p_{1}, p_{3}^{\prime}\right)=\varnothing$ and $\gamma\left(r_{2} ; p_{3}, p_{1}^{\prime}\right)=\varnothing$, we have $\left(v_{4} v_{5} p_{4}\right)_{3},\left(r_{3} p_{5} v_{1} r_{2} p_{3}\right)_{5}$ and a 4-hole from the remaining points.

Case $3|V(P)|=4$.

Let $v_{i} \in V(P)$ for $i=1,2,3,4$. We have 4 friend points $p_{i}$ for $i=1,2,3,4$. Then we have 5 points $r_{1}, r_{2}, r_{3}, r_{4}, r_{5}$ remained as shown in Figure 6 . 


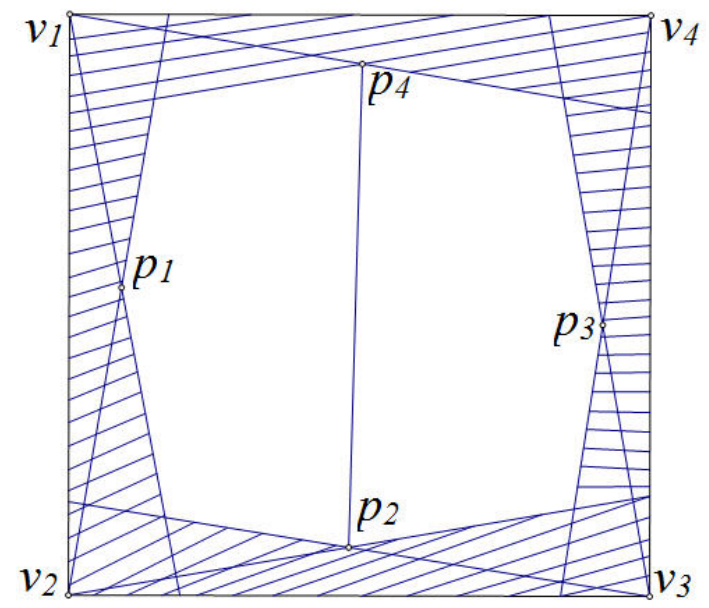

Figure 6. Figure of $|\mathrm{V}(\mathrm{P})|=4$.

If $\gamma\left(p_{1} ; v_{1}^{\prime}, v_{2}^{\prime}\right) \cap H\left(p_{1} ; p_{2} p_{4}\right)=\varnothing$ or $\gamma\left(p_{3} ; v_{3}^{\prime}, v_{4}^{\prime}\right) \cap H\left(p_{3} ; p_{2} p_{4}\right)=\varnothing$, we have a cutting line $L_{5}\left(p_{4}, \alpha\left(p_{4} ; p_{2}, v_{1}^{\prime}\right)\right)$ or $L_{5}\left(p_{4}, \alpha\left(p_{4} ; p_{2}, v_{4}^{\prime}\right)\right)$. Then we will consider that $\gamma\left(p_{1} ; v_{1}^{\prime}, v_{2}^{\prime}\right) \cap$ $H\left(p_{1} ; p_{2} p_{4}\right) \neq \varnothing$ and $\gamma\left(p_{3} ; v_{3}^{\prime}, v_{4}^{\prime}\right) \cap H\left(p_{3} ; p_{2} p_{4}\right) \neq \varnothing$.

Assume one of the five points say $r_{1} \in \gamma\left(p_{1} ; v_{1}^{\prime}, v_{2}^{\prime}\right) \cap H\left(p_{1} ; p_{2} p_{4}\right)$ and the remaining four say $r_{i} \in \gamma\left(p_{3} ; v_{3}^{\prime}, v_{4}^{\prime}\right) \cap H\left(p_{3} ; p_{2} p_{4}\right), i=2,3,4,5$. (If $\gamma\left(p_{1} ; v_{1}^{\prime}, v_{2}^{\prime}\right) \cap H\left(p_{1} ; p_{2} p_{4}\right)$ has four points and $\gamma\left(p_{1} ; v_{1}^{\prime}, v_{2}^{\prime}\right) \cap H\left(p_{1} ; p_{2} p_{4}\right)$ has one point, symmetrically, the conclusion is also right). Let $r_{2}=\alpha\left(p_{4} ; p_{2}, v_{1}^{\prime}\right)$.

Suppose $r_{1} \in \gamma\left(p_{1} ; v_{1}^{\prime}, p_{2}\right)$ or $r_{1} \in \gamma\left(p_{1} ; v_{2}^{\prime}, p_{4}\right)$. We always have a cutting line $L_{5}\left(p_{2}, p_{4}\right)$. Suppose $\left.r_{1} \in \gamma\left(p_{1} ; p_{4}, r_{2}\right) \cap H\left(p_{1} ; p_{2} p_{4}\right)\right)$. We have $\left(v_{1} v_{4} p_{4}\right)_{3},\left(p_{1} v_{2} p_{2} r_{2} r_{1}\right)_{5}$ and a 4 -hole from the remaining points. Suppose $r_{1} \in \gamma\left(p_{1} ; p_{2}, r_{2}\right) \cap H\left(p_{1} ; p_{2} p_{4}\right)$. We have $\left(v_{2} v_{3} p_{2}\right)_{3},\left(p_{1} v_{1} p_{4} r_{2} r_{1}\right)_{5}$ and a 4-hole from the remaining points.

Assume two of the five points, say $r_{1}, r_{2} \in \gamma\left(p_{1} ; v_{1}^{\prime}, v_{2}^{\prime}\right) \cap H\left(p_{1} ; p_{2} p_{4}\right)$ and the remaining three say $r_{i} \in \gamma\left(p_{3} ; v_{3}^{\prime}, v_{4}^{\prime}\right) \cap H\left(p_{3} ; p_{2} p_{4}\right), i=3,4,5$. (If $\gamma\left(p_{1} ; v_{1}^{\prime}, v_{2}^{\prime}\right) \cap H\left(p_{1} ; p_{2} p_{4}\right)$ has three points and $\gamma\left(p_{1} ; v_{1}^{\prime}, v_{2}^{\prime}\right) \cap H\left(p_{1} ; p_{2} p_{4}\right)$ has two points, symmetrically, our conclusion is also right.)

Suppose $\gamma\left(p_{2} ; v_{1}, p_{4}\right)=\varnothing$. If $\gamma\left(p_{2} ; v_{1}, p_{1}\right) \neq \varnothing$, let $r_{1}=\alpha\left(p_{2} ; v_{1}, p_{1}\right)$, we have $\left(r_{2} p_{1} v_{2}\right)_{3}$, $\left(p_{4} v_{1} r_{1} p_{2} \alpha\left(p_{2} ; p_{4}, v_{2}^{\prime}\right)\right)_{5}$ and a 4-hole from the remaining points. If $\gamma\left(p_{2} ; v_{1}, p_{1}\right)=\varnothing$, we have $\left(r_{1} r_{2} v_{2}\right)_{3},\left(p_{4} v_{1} p_{1} p_{2} \alpha\left(p_{2} ; p_{4}, v_{2}^{\prime}\right)\right)_{5}$ and a 4-hole from the remaining points. Suppose $\gamma\left(p_{2} ; v_{1}, p_{4}\right) \neq$ $\varnothing$. Let $r_{1}=\alpha\left(p_{2} ; p_{4}, v_{1}\right)$. If $r_{2} \in \gamma\left(r_{1} ; p_{1}, p_{2}^{\prime}\right)$, we have $\left(v_{1} v_{4} p_{4}\right)_{3},\left(r_{1} r_{2} p_{1} v_{2} p_{2}\right)_{5}$ and a 4-hole from the remaining points. If $r_{2} \in \gamma\left(r_{1} ; p_{1}, p_{4}^{\prime}\right)$, we have $\left(v_{2} p_{2} v_{3}\right)_{3},\left(v_{1} p_{1} r_{2} r_{1} p_{4}\right)_{5}$ and a 4-hole from the remaining points. If $r_{2} \in \gamma\left(r_{1} ; p_{2}, p_{4}^{\prime}\right)$, we have $\left(v_{1} v_{2} p_{1}\right)_{3},\left(p_{4} r_{1} r_{2} p_{2} \alpha\left(p_{2} ; p_{4}, v_{2}^{\prime}\right)_{5}\right.$ and a 4-hole from the remaining points.

Case $4|V(P)|=3$.

Let $v_{1}, v_{2}, v_{3} \in V(P)$. We have 3 friend points $p_{1}, p_{2}, p_{3}$ and 7 points remained. As shown in Figure 7 , denote $\gamma\left(p_{1} ; v_{2}^{\prime}, p_{3}\right) \cap \gamma\left(p_{3} ; v_{3}^{\prime}, p_{1}\right)=T_{1}, \gamma\left(p_{1} ; v_{1}^{\prime}, p_{2}\right) \cap \gamma\left(p_{2} ; v_{3}^{\prime}, p_{1}\right)=T_{2}, \gamma\left(p_{2} ; v_{2}^{\prime}, p_{3}\right) \cap$ $\gamma\left(p_{3} ; v_{1}^{\prime}, p_{1}\right)=T_{3}$.

Without loss of generality, we assume $\left|T_{3}\right| \geq\left|T_{1}\right| \geq\left|T_{2}\right|$.

(1) $\left|T_{3}\right|=7$.

We have a cutting line $\left.L_{5}\left(p_{2}, \alpha\left(p_{2} ; p_{3}, v_{2}^{\prime}\right)\right)\right)$.

(2) $\left|T_{3}\right|=6$.

Name the remaining one $r_{1}$. If $r_{1} \in \gamma\left(p_{3} ; v_{3}^{\prime}, p_{1}\right)$ or $r_{1} \in \gamma\left(p_{2} ; v_{3}^{\prime}, p_{1}\right)$, we have a cutting line $L_{5}\left(p_{2}, p_{3}\right)$. If $r_{1} \in \gamma\left(p_{3} ; p_{1}, p_{2}\right) \cap \gamma\left(p_{1} ; p_{2}, p_{3}\right):$ and if $\gamma\left(r_{1} ; p_{3}, p_{1}^{\prime}\right) \neq \varnothing$, we have a cutting line 
$L_{5}\left(r_{1}, \alpha\left(r_{1} ; p_{3}, p_{1}^{\prime}\right)\right)$; and if $\gamma\left(r_{1} ; p_{3}, p_{1}^{\prime}\right)=\varnothing$, we have $\left(v_{1} v_{3} p_{3}\right)_{3},\left(r_{1} p_{1} v_{2} p_{2} \alpha\left(r_{1} ; p_{2}, p_{1}^{\prime}\right)\right)_{5}$ and a 4-hole from the remaining points.

(3) $\left|T_{3}\right|=\mathbf{5}$

Name the remaining two points $r_{1}, r_{2}$. Then we will discuss the region $\gamma\left(p_{3} ; v_{1}, p_{1}\right)$, as shown in Figure 8.

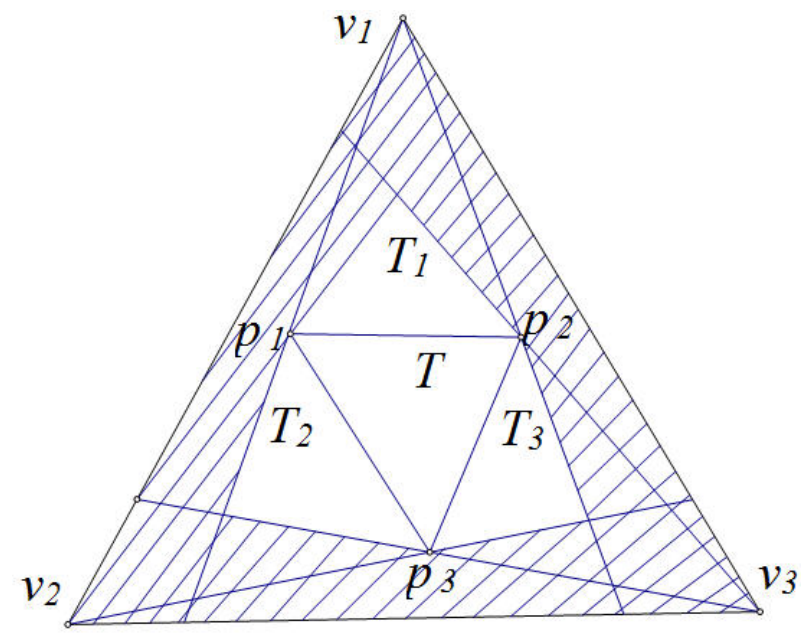

Figure 7. Figure of $|\mathrm{V}(\mathrm{P})|=5$.

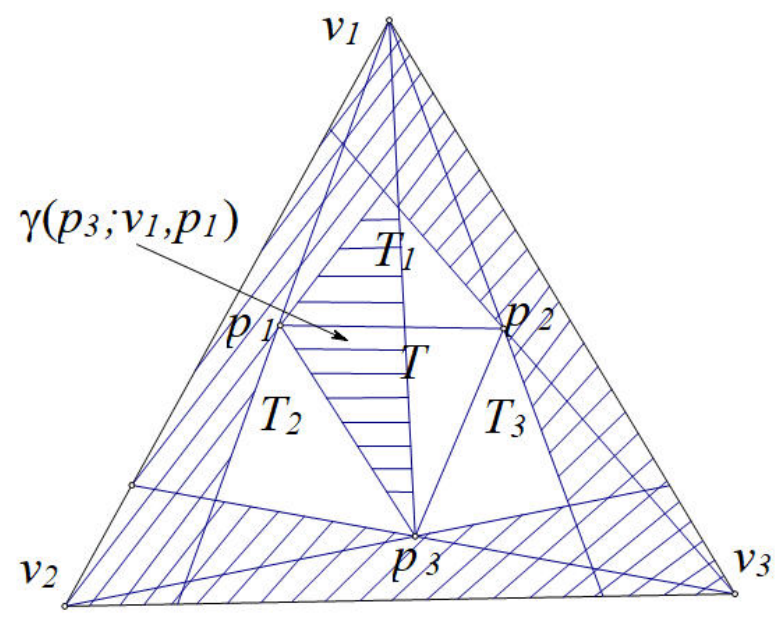

Figure 8. Figure of $\left|T_{3}\right|=5$

Assume $\gamma\left(p_{3} ; v_{1}, p_{1}\right)=\varnothing$. (If $\gamma\left(p_{1} ; p_{2}, v_{2}\right)=\varnothing$, by the similar reason our conclusion is also right.) Let $r_{1}=\alpha\left(p_{3} ; p_{1}, p_{2}\right)$. Suppose $r_{1} \in \gamma\left(p_{2} ; p_{1}, p_{3}\right)$.

If $r_{2} \in \gamma\left(r_{1} ; p_{3}, p_{1}^{\prime}\right)$, we have a cutting line $L_{5}\left(p_{3}, r_{2}\right)$. If $r_{2} \in \gamma\left(p_{2} ; r_{1}, p_{1}\right)$ : and if $\gamma\left(r_{1} ; p_{3}, p_{1}^{\prime}\right) \neq$ $\varnothing$, we have $\left(r_{2} p_{2} v_{2}\right)_{3},\left(p_{3} v_{1} p_{1} r_{1} \alpha\left(r_{1} ; p_{3}, p_{1}^{\prime}\right)\right)_{5}$ and a 4-hole from the remaining points; and if $\gamma\left(r_{1} ; p_{3}, p_{1}^{\prime}\right)=\varnothing$, we have $\left(v_{1} v_{2} p_{1}\right)_{3},\left(p_{3} r_{1} r_{2} p_{2} \alpha\left(p_{2} ; p_{3}, v_{3}\right)\right)_{5}$ and a 4 -hole from the remaining points. Suppose $r_{1} \in \gamma\left(p_{2} ; p_{1}, v_{3}^{\prime}\right)$. If $r_{2} \in \gamma\left(r_{1} ; p_{3}, p_{1}^{\prime}\right)$, we have a cutting line $L_{5}\left(p_{3}, r_{2}\right)$. If $r_{2} \in \gamma\left(r_{1} ; p_{1}^{\prime}, p_{3}^{\prime}\right)$, we have $\left(r_{1} v_{2} p_{2}\right)_{3},\left(p_{3} v_{1} p_{1} r_{1} \alpha\left(r_{1} ; p_{3}, p_{2}\right)\right)_{5}$ and a 4 -hole from the remaining points.

Assume $\gamma\left(p_{3} ; v_{1}, p_{1}\right) \neq \varnothing$ and $\gamma\left(p_{1} ; p_{2}, v_{2}\right) \neq \varnothing$. Then we suppose $\gamma\left(p_{3} ; v_{1}, p_{1}\right)$ has one point say $r_{1}$ and $\gamma\left(p_{1} ; p_{2}, v_{2}\right)$ has one point say $r_{2}$. If $\gamma\left(r_{1} ; p_{1}, p_{2}^{\prime}\right) \neq \varnothing$, we have a cutting line $L_{5}\left(p_{2}, r_{1}\right)$. If $\gamma\left(r_{1} ; p_{1}, p_{2}^{\prime}\right)=\varnothing$, we have a cutting line $L_{5}\left(r_{1}, \alpha\left(r_{1} ; p_{2}, p_{3}\right)\right.$.

(4) $\left|T_{3}\right|=4$. 
Name the remaining three points $r_{1}, r_{2}, r_{3}$. Then we will discuss the region $\gamma\left(p_{3} ; p_{1}, v_{3}^{\prime}\right)$, as shown in Figure 9.

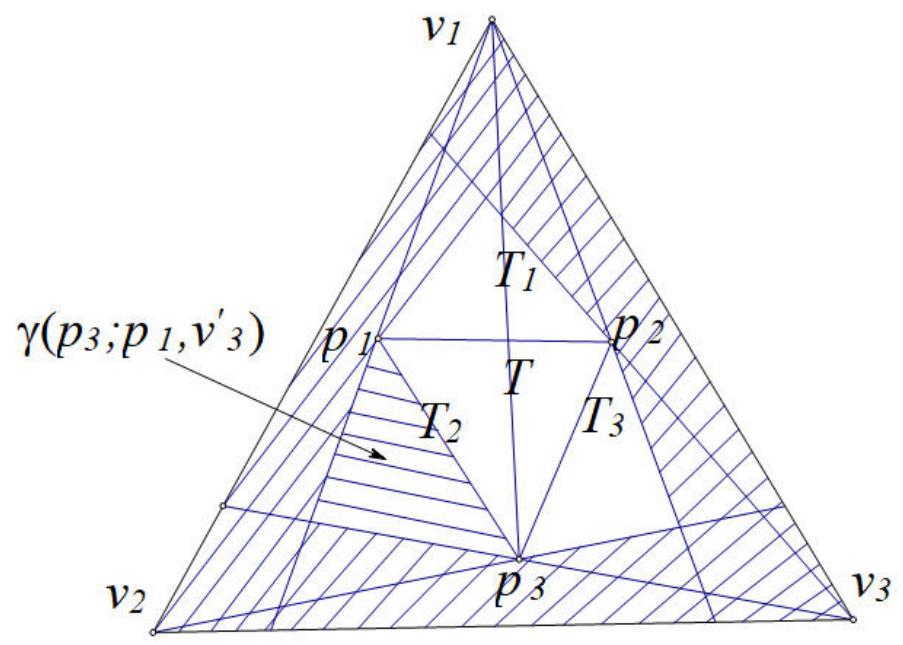

Figure 9. Figure of $\left|T_{3}\right|=4$

(a) Assume $r_{1}, r_{2}, r_{3} \in \gamma\left(p_{3} ; p_{1}, v_{3}^{\prime}\right)$. Let $r_{1}=\alpha\left(p_{3} ; p_{1}, v_{3}^{\prime}\right)$. We have $\left(v_{1} p_{2} p_{3}\right)_{3},\left(r_{1} p_{1} v_{2} p_{2} p_{3}\right)_{5}$ and a 4-hole from the remaining points.

(b) Assume two of $r_{i}, i=1,2,3$, say $r_{1}, r_{2} \in \gamma\left(p_{3} ; p_{1}, v_{3}^{\prime}\right)$. Suppose $r_{3} \in \gamma\left(p_{2} ; p_{1}, p_{3}\right) \cap$ $\gamma\left(p_{3} ; p_{1}, p_{2}\right)$. If $\gamma\left(r_{3} ; p_{1}, p_{2}^{\prime}\right) \neq \varnothing$ : we have a 4-hole from $\left\{r_{4}, r_{5}, r_{6}, r_{7}, v_{3}\right\}$, $\left(p_{1} v_{2} p_{2} r_{3} \alpha\left(r_{3} ; p_{1}, p_{2}^{\prime}\right)\right)_{5}$ and a 3 -hole from the remaining points. If $\gamma\left(r_{3} ; p_{1}, p_{2}^{\prime}\right) \neq \varnothing$, we have $\left(r_{3} p_{1} v_{2} p_{2} \alpha\left(r_{3} ; p_{2}, p_{1}^{\prime}\right)\right)_{5}, \quad\left(r_{1} r_{2} v_{1}\right)_{3}$ and a 4-hole from the remaining points. If $\gamma\left(r_{3} ; p_{1}, p_{2}^{\prime}\right)=\varnothing$ and $\gamma\left(r_{3} ; p_{1}, p_{2}^{\prime}\right)=\varnothing$, we have a cutting line $L_{4}\left(p_{2}, r_{3}\right)$.

(c) Assume one of $r_{i}, i=1,2,3$, say $r_{1} \in \gamma\left(p_{3} ; p_{1}, v_{3}^{\prime}\right)$.

Suppose $\gamma\left(r_{3} ; p_{1}, v_{2}\right)=\varnothing$. We have a cutting line $L_{5}\left(p_{3}, r_{2}\right)$.

Suppose $\gamma\left(p_{3} ; p_{1}, v_{2}\right) \neq \varnothing$. Let $r_{2}=\alpha\left(p_{3} ; p_{1}, v_{2}\right)$. If $r_{2} \in \gamma\left(p_{1} ; v_{1}^{\prime}, p_{2}\right)$, we have a cutting line $L_{5}\left(r_{2}, \alpha\left(r_{2} ; p_{3}, p_{2}\right)\right)$. Then we suppose $r_{2} \in \gamma\left(p_{1} ; p_{2}, p_{3}\right)$. If $r_{1} \in \gamma\left(r_{2} ; p_{2}^{\prime}, p_{1}\right)$ : and if $r_{3} \in \gamma\left(r_{2} ; p_{3}, p_{1}^{\prime}\right)$, we have a cutting line $L_{5}\left(r_{2}, r_{3}\right)$; and if $r_{3} \in \gamma\left(r_{2} ; p_{2}, p_{1}^{\prime}\right)$, we have $\left(v_{1} r_{1} p_{3}\right)_{3}$, $\left(p_{1} v_{2} p_{2} r_{3} r_{2}\right)_{5}$ and a 4 -hole from the remaining points; and if $r_{3} \in \gamma\left(r_{2} ; p_{2}, v_{2}\right)$, we have $\left(v_{1} v_{3} p_{3}\right)_{3},\left(r_{1} p_{1} v_{2} r_{3} r_{2}\right)_{5}$ and a 4-hole from the remaining points; and if $r_{3} \in \gamma\left(r_{2} ; v_{2}, p_{3}^{\prime}\right)$, we have a cutting line $L_{5}\left(v_{2}, p_{3}\right)$. If $r_{1} \in \gamma\left(r_{2} ; p_{2}^{\prime}, p_{3}\right)$ : and if $r_{3} \in \gamma\left(r_{2} ; p_{3}, p_{1}^{\prime}\right)$, we have a cutting line $L_{5}\left(r_{2}, \alpha\left(r_{2} ; p_{3}, p_{1}^{\prime}\right)\right)$; and if $r_{3} \in \gamma\left(r_{2} ; p_{2}, p_{1}^{\prime}\right)$, we have $\left(v_{1} r_{1} p_{3}\right)_{3},\left(r_{2} p_{1} v_{2} p_{2} r_{3}\right)_{5}$ and a 4-hole from the remaining points; and if $r_{3} \in \gamma\left(r_{2} ; r_{1}^{\prime}, p_{2}\right)$, we have $\left(v_{1} v_{2} p_{1}\right)_{3},\left(p_{3} r_{1} r_{2} r_{3} p_{2}\right)_{5}$ and a 4-hole from the remaining points; and if $r_{3} \in \gamma\left(r_{2} ; v_{2}, r_{1}^{\prime}\right)$, we have $\left(v_{1} v_{3} p_{3}\right)_{3},\left(r_{1} p_{1} v_{2} r_{3} r_{2}\right)_{5}$ and a 4-hole from the remaining points; and if $r_{3} \in \gamma\left(r_{2} ; p_{3}^{\prime}, v_{2}\right)$, we have $\left(v_{1} r_{1} p_{1}\right)_{3},\left(p_{3} r_{2} r_{3} v_{2} p_{2}\right)_{5}$ and a 4-hole from the remaining points.

(d) Assume $\gamma\left(p_{3} ; p_{1}, v_{3}^{\prime}\right)=\varnothing$. By the same reason, we also assume $\gamma\left(p_{1} ; p_{2}, v_{1}^{\prime}\right)=\varnothing$. Then we will discuss the region $\gamma\left(v_{1} ; p_{1}, p_{2}\right)$, as shown in Figure 10. 


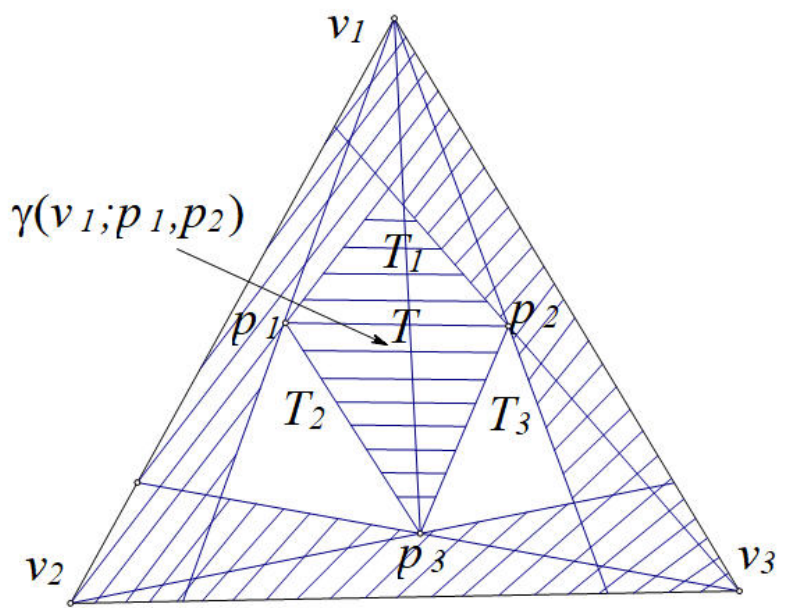

Figure 10. Figure of $\left|T_{3}\right|=4$ with shaded region nonempty.

(d1) Suppose $\gamma\left(v_{1} ; p_{1}, p_{2}\right)=\varnothing$. Let $r_{1}=\alpha\left(p_{1} ; p_{3}, p_{2}\right)$ within $\left(p_{1} p_{2} p_{3}\right)$.

If $\gamma\left(r_{1} ; p_{1}, p_{3}^{\prime}\right) \neq \varnothing$, we have $\left(v_{2} v_{3} p_{2}\right)_{3},\left(r_{1} p_{3} v_{1} p_{1} \alpha\left(r_{1} ; p_{1}, p_{3}^{\prime}\right)\right)_{5}$ and a 4-hole from the remaining points.

If $\gamma\left(r_{1} ; p_{1}, p_{3}^{\prime}\right)=\varnothing$ : and if $\gamma\left(r_{1} ; p_{3}, p_{1}^{\prime}\right) \neq \varnothing$, we have $\left(v_{2} v_{3} p_{2}\right)_{3}$, $\left(p_{3} v_{1} p_{1} r_{1} \alpha\left(r_{1} ; p_{3}, p_{1}\right)\right)_{5}$ and a 4-hole from the remaining points; and if $\gamma\left(r_{1} ; p_{3}, p_{1}^{\prime}\right)=\varnothing$, let $r_{2}=\alpha\left(r_{1} ; p_{3}^{\prime}, p_{1}^{\prime}\right)$ within $\left(p_{1} p_{2} p_{3}\right)$, we have $\left(v_{1} v_{3} p_{3}\right)_{3},\left(r_{1} p_{1} v_{2} r_{2} r_{3}\right)_{5}$ and a 4 -hole from the remaining points when $r_{3} \in \gamma\left(r_{2} ; r_{1}, v_{2}^{\prime}\right) \cap \gamma\left(r_{1} ; r_{2}, p_{1}^{\prime}\right)$, we have $\left(v_{1} p_{1} r_{1} p_{3}\right)_{4}$, $\left(r_{3} r_{2} v_{2} p_{2} \alpha\left(r_{3} ; p_{2}, r_{2}\right)\right)_{5}$ and a 3-hole from the remaining points when $r_{3} \in \gamma\left(r_{2} ; p_{1}^{\prime}, v_{2}^{\prime}\right)$ and $\gamma\left(p_{3} ; p_{2}, v_{1}^{\prime}\right) \cap \gamma\left(r_{3} ; p_{2}, r_{2}^{\prime}\right) \neq \varnothing$, we have $\left(v_{1} v_{2} p_{1}\right)_{3},\left(p_{3} r_{1} r_{2} r_{3} \alpha\left(r_{3} ; p_{3}, r_{2}^{\prime}\right)\right)_{5}$ and a 4-hole from the remaining points when $r_{3} \in \gamma\left(r_{2} ; p_{1}^{\prime}, v_{2}^{\prime}\right)$ and $\gamma\left(p_{3} ; p_{2}, v_{1}^{\prime}\right) \cap$ $\gamma\left(r_{3} ; p_{2}, r_{2}^{\prime}\right)=\varnothing$, we have $\left(v_{1} r_{1} p_{3}\right)_{3},\left(p_{1} v_{2} p_{2} r_{3} r_{2}\right)_{5}$ and a 4-hole from the remaining points when $r_{3} \in \gamma\left(r_{2} ; p_{1}^{\prime}, p_{2}\right)$, we have $\left(v_{1} v_{2} p_{1}\right)_{3},\left(p_{3} r_{1} r_{2} r_{3} p_{2}\right)_{5}$ and a 4-hole from the remaining points when $r_{3} \in \gamma\left(r_{2} ; r_{1}^{\prime}, p_{2}\right)$.

(d2) Suppose $\gamma\left(v_{1} ; p_{1}, p_{2}\right)$ has one of the $r_{1}, r_{2}, r_{3}$, say $r_{1} \in \gamma\left(r_{1} ; p_{1}, p_{2}\right)$. Let $r_{2}=\alpha\left(p_{2} ; p_{1}, p_{3}\right)$. If $r_{2} \in \gamma\left(r_{1} ; p_{2}, p_{3}\right)$, we have $\left(v_{2} v_{3} p_{2}\right)_{3},\left(r_{1} p_{1} v_{1} p_{3} r_{2}\right)_{5}$ and a 4 -hole from the remaining points.

If $r_{2} \in \gamma\left(r_{1} ; p_{1}, p_{3}\right):$ and if $r_{3} \in \gamma\left(r_{2} ; r_{1}, p_{3}\right)$, we have $\left(v_{1} v_{2} p_{1}\right)_{3},\left(r_{3} r_{2} r_{1} p_{2} p_{3}\right)_{5}$ and a 4 -hole from the remaining points; and if $r_{3} \in \gamma\left(r_{2} ; p_{3}, p_{1}^{\prime}\right)$, we have $\left(v_{2} v_{3} p_{2}\right)_{3}$, $\left(v_{1} p_{1} r_{2} r_{3} p_{3}\right)_{5}$ and a 4 -hole from the remaining points; and if $r_{3} \in \gamma\left(r_{2} ; p_{1}^{\prime}, v_{1}^{\prime}\right)$, we have a cutting line $L_{5}\left(r_{1}, \alpha\left(r_{1} ; p_{2}, p_{1}^{\prime}\right)\right)$ when $\gamma\left(r_{1} ; p_{2}, p_{1}^{\prime}\right) \neq \varnothing$, we have $\left(v_{2} v_{3} p_{2}\right)_{3}$, $\left(r_{3} r_{2} p_{1} r_{1} \alpha\left(r_{3} ; r_{1}, r_{2}^{\prime}\right)\right)_{5}$ and a 4-hole from the remaining points when $\gamma\left(r_{1} ; p_{2}, p_{1}^{\prime}\right)=\varnothing$ and $\gamma\left(r_{3} ; r_{1}, r_{2}^{\prime}\right) \neq \varnothing$, we have $\left(r_{1} p_{1} v_{2} p_{2}\right)_{4},\left(p_{3} v_{1} r_{2} r_{3} \alpha\left(r_{3} ; p_{3}, r_{2}^{\prime}\right)\right)_{5}$ and a 3-hole from the remaining points when $\gamma\left(r_{1} ; p_{2}, p_{1}^{\prime}\right)=\varnothing$ and $\gamma\left(r_{3} ; r_{1}, r_{2}^{\prime}\right)=\varnothing$.

If $r_{2} \in \gamma\left(r_{1} ; p_{2}, p_{3}\right)$, we have $\left(v_{2} v_{3} p_{2}\right)_{3},\left(p_{3} v_{1} p_{1} r_{1} r_{2}\right)_{5}$ and a 4 -hole from the remaining points.

(d3) Suppose $\gamma\left(v_{1} ; p_{1}, p_{2}\right)$ has two of the points $r_{1}, r_{2}, r_{3}$, say $r_{1}, r_{2} \in \gamma\left(r_{1} ; p_{1}, p_{2}\right)$. Let $r_{1}=\alpha\left(p_{2} ; p_{1}, p_{3}\right)$.

If $\gamma\left(r_{1} ; p_{2}, p_{1}^{\prime}\right) \neq \varnothing$, we have a cutting line $L_{5}\left(r_{1}, \alpha\left(r_{1} ; p_{2}, p_{1}^{\prime}\right)\right)$.

If $\gamma\left(r_{1} ; p_{2}, p_{1}^{\prime}\right)=\varnothing$, let $r_{2}=\alpha\left(p_{1} ; p_{2}, p_{2}\right)$ : and if $r_{2} \in \gamma\left(v_{1} ; p_{2}, p_{3}\right)$, we have a cutting line $L_{5}\left(r_{2}, r_{3}\right)$ when $r_{3} \in \gamma\left(r_{2} ; p_{1}, p_{3}\right)$, we have $\left(v_{1} p_{1} v_{2}\right)_{3},\left(p_{3} r_{2} r_{3} r_{1} p_{2}\right)_{5}$ and a 4-hole from the remaining points when $r_{3} \in \gamma\left(r_{2} ; p_{3}, r_{1}\right)$, we have $\left(v_{2} v_{3} p_{2}\right)_{3},\left(r_{2} v_{1} p_{1} r_{1} p_{3}\right)_{5}$ 
and a 4-hole from the remaining points when $r_{3} \in \gamma\left(r_{1} ; r_{2}, p_{1}^{\prime}\right)$, we have $\left(v_{1} p_{3} v_{3}\right)_{3}$, $\left(r_{1} p_{1} v_{2} p_{2} r_{3}\right)_{5}$ and a 4-hole from the remaining points when $r_{3} \in \gamma\left(r_{1} ; p_{2}, p_{1}^{\prime}\right)$; and if $r_{2} \in \gamma\left(v_{1} ; p_{1}, p_{2}\right)$, we have $\left(p_{1} r_{1} p_{2} v_{2}\right)_{4},\left(p_{1} v_{1} r_{1} r_{3} \alpha\left(r_{3} ; p_{3}, r_{2}^{\prime}\right)\right)_{5}$ and a 3-hole from the remaining points when $\gamma\left(r_{2} ; r_{3}, p_{1}^{\prime}\right) \cap \gamma\left(p_{2} ; p_{3}, v_{2}^{\prime}\right) \neq \varnothing$, we have a cutting line $L_{5}\left(r_{1}, \alpha\left(r_{1} ; p_{2}, p_{1}^{\prime}\right)\right)$, when $\gamma\left(r_{1} ; p_{2}, p_{1}^{\prime}\right) \neq \varnothing$, we have $\left(v_{1} v_{3} p_{3}\right)_{3},\left(r_{3} r_{2} p_{1} r_{1} \alpha\left(r_{1} ; r_{3}, p_{1}^{\prime}\right)\right)_{5}$ and a 4-hole from the remaining points when $\gamma\left(r_{2} ; r_{3}, p_{1}^{\prime}\right) \cap \gamma\left(p_{2} ; p_{3}, v_{2}^{\prime}\right)=\varnothing$ and $\gamma\left(r_{1} ; p_{2}, p_{1}^{\prime}\right)=\varnothing$.

(d4) Suppose $\gamma\left(v_{1} ; p_{1}, p_{2}\right)$ has all of the three points $r_{1}, r_{2}, r_{3}$. Let $r_{1}=\alpha\left(p_{1} ; p_{3}, p_{2}\right)$, $r_{2}=\alpha\left(p_{1} ; p_{2}, p_{3}\right)$.

If $\gamma\left(r_{1} ; p_{3}, p_{1}^{\prime}\right) \neq \varnothing$ or $\gamma\left(r_{2} ; p_{2}, p_{1}^{\prime}\right) \neq \varnothing$, we always have a cutting line $L_{5}$.

If $\gamma\left(r_{1} ; p_{3}, p_{1}^{\prime}\right)=\varnothing$ and $\gamma\left(r_{2} ; p_{2}, p_{1}^{\prime}\right)=\varnothing$ : and if $r_{3} \in \gamma\left(r_{1} ; p_{1}, p_{3}^{\prime}\right)$, we have a cutting line $L_{5}\left(p_{3}, r_{1}\right)$; and if $r_{3} \in \gamma\left(r_{1} ; p_{3}^{\prime}, p_{2}\right) \cap \gamma\left(r_{2} ; p_{2}^{\prime}, r_{1}\right)$, we have $\left(v_{1} v_{2} p_{1}\right)_{3},\left(p_{3} r_{1} r_{3} r_{2} p_{2}\right)_{5}$ and a 4-hole from the remaining points; and if $r_{3} \in \gamma\left(r_{2} ; p_{1}, p_{2}^{\prime}\right)$, we have a cutting line $L_{5}\left(p_{2}, r_{2}\right)$; and if $r_{3} \in \gamma\left(r_{2} ; p_{1}^{\prime}, v_{2}^{\prime}\right)$, we have $\left(v_{1} p_{1} r_{1} p_{3}\right)_{4},\left(r_{3} r_{2} v_{2} p_{2} \alpha\left(r_{3} ; p_{2}, r_{2}^{\prime}\right)\right)_{5}$ and a 3-hole from the remaining points when $\gamma\left(r_{3} ; p_{2}, r_{2}^{\prime}\right) \cap \gamma\left(v_{1} ; p_{2}, p_{3}\right) \neq \varnothing$, we have $\left(v_{2} v_{3} p_{2}\right)_{3},\left(r_{1} p_{1} r_{2} r_{3} \alpha\left(r_{3} ; p_{3}, r_{2}^{\prime}\right)\right)_{5}$ and a 4-hole from the remaining points when $\gamma\left(r_{3} ; p_{2}, r_{2}^{\prime}\right) \cap \gamma\left(v_{1} ; p_{2}, p_{3}\right) \neq \varnothing$.

(5) $\left|T_{3}\right|=3$. Let $r_{1}, r_{2}, r_{3} \in T_{3}$.

(a) $\left|T_{1}\right|=3$.

Let $r_{4}, r_{5}, r_{6} \in T_{1}$. Name the remaining one point $r_{7}$. Assume $r_{7} \in \gamma\left(v_{2} ; p_{3}, p_{2}\right)$, as shown in Figure 11.

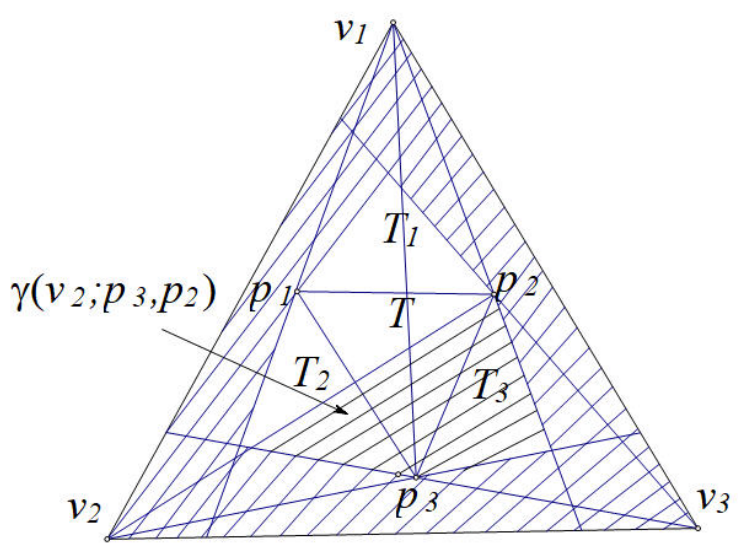

Figure 11. Figure of $\left|\mathrm{T}_{1}\right|=3$.

Symmetrically, our conclusion is also right when $r_{7} \in \gamma\left(v_{2} ; p_{3}, p_{1}\right)$. Let $r_{4}=\alpha\left(p_{3} ; p_{1}, v_{3}^{\prime}\right)$. We have $\left(r_{5} r_{6} v_{1}\right)_{3},\left(r_{4} p_{1} v_{2} r_{7} p_{3}\right)_{5}$ and a 4 -hole from the remaining points.

(b) $\quad\left|T_{1}\right|=2$.

Let $r_{4}, r_{5} \in T_{1}$. Name the remaining two points $r_{6}, r_{7}$.

(b1) $\left|T_{2}\right|=2$. Let $r_{6}, r_{7} \in T_{2}$.

Assume $\gamma\left(v_{1} ; p_{1}, p_{2}\right)=\varnothing$. Let $r_{4}=\alpha\left(p_{2} ; v_{1}, p_{3}\right)$. Suppose $r_{5} \in \gamma\left(r_{4} ; p_{2}^{\prime}, p_{3}\right)$. We have a cutting line $L_{5}\left(p_{1}, p_{3}\right)$. Suppose $r_{5} \in \gamma\left(r_{4} ; v_{1}^{\prime}, p_{3}\right)$. If $\gamma\left(r_{5} ; p_{3}, r_{4}^{\prime}\right) \neq \varnothing$, we have a cutting line $L_{5}\left(r_{5}, \alpha\left(r_{5} ; p_{3}, v_{4}^{\prime}\right)\right)$. If $\gamma\left(r_{5} ; p_{3}, r_{4}^{\prime}\right)=\varnothing$, we have a cutting line $L_{5}\left(r_{1}, \alpha\left(r_{1} ; p_{1}, p_{2}\right)\right.$ where $r_{1}=\alpha\left(p_{1} ; p_{3}, p_{2}\right)$. Suppose $r_{5} \in \gamma\left(r_{4} ; p_{2}^{\prime}, v_{1}^{\prime}\right)$. We have $\left(r_{6} r_{7} v_{2}\right)_{3},\left(r_{4} v_{1} p_{1} p_{2} r_{5}\right)_{5}$ and a 4 -hole from the remaining points. 
Assume $\gamma\left(v_{1} ; p_{1}, p_{2}\right)$ has one of $r_{4}, r_{5}$. Let $r_{4} \in \alpha\left(v_{1} ; p_{1}, p_{2}\right)$. Suppose $r_{5} \in \alpha\left(r_{4} ; p_{1}^{\prime}, v_{1}\right)$. If $\gamma\left(r_{4} ; p_{2}, v_{1}^{\prime}\right)=\varnothing$, we have $\left(r_{2} r_{3} v_{3}\right)_{3},\left(r_{5} r_{4} p_{2} r_{1} p_{3}\right)_{5}$ and a 4-hole from the remaining points where $r_{1}=\alpha\left(p_{2} ; p_{3}, v_{3}\right)$. If $\gamma\left(r_{4} ; p_{2}, v_{1}^{\prime}\right) \neq \varnothing$, we have $\left(p_{1} v_{2} r_{7}\right)_{3},\left(v_{1} r_{4} r_{6} p_{2} r_{5}\right)_{5}$ and a 4-hole from the remaining points where $r_{6}=\alpha\left(r_{4} ; p_{2}, v_{1}^{\prime}\right)$.

Assume $\gamma\left(v_{1} ; p_{1}, p_{2}\right)$ has $r_{4}, r_{5}$. Let $r_{4} \in \alpha\left(p_{2} ; v_{1}, p_{1}\right), r_{1}=\alpha\left(p_{2} ; p_{3}, v_{3}\right)$. we have $\left(r_{2} r_{3} v_{3}\right)_{3},\left(p_{2} r_{1} p_{3} v_{1} r_{4}\right)_{5}$ and a 4 -hole from the remaining points.

(b2) $\quad\left|T_{2}\right|=1$.

Let $r_{6} \in T_{2}$ and $r_{7} \in\left(p_{1} p_{2} p_{3}\right)$, as shown in Figure 12 .

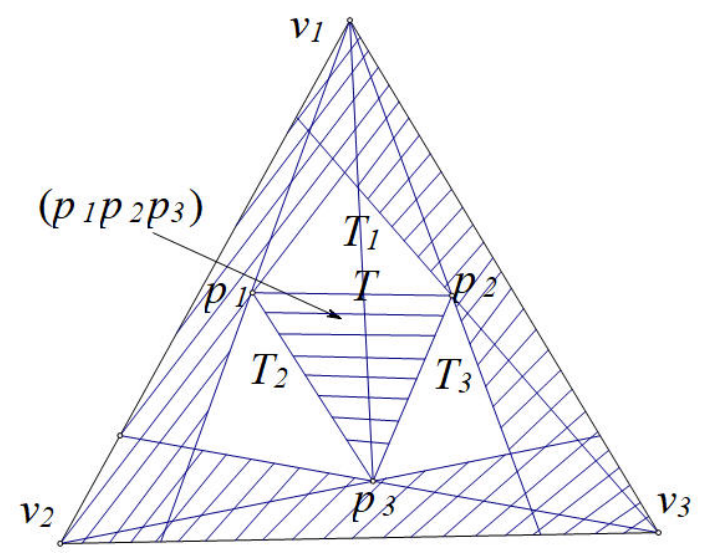

Figure 12. Figure of $\left|T_{2}\right|=1$.

Assume $r_{6} \in \gamma\left(r_{7} ; p_{3}^{\prime}, p_{2}\right)$. We have $\left(r_{2} r_{3} v_{3}\right)_{3},\left(p_{3} r_{7} r_{6} p_{2} r_{1}\right)_{5}$ and a 4-hole from the remaining points where $r_{1}=\alpha\left(p_{2} ; p_{3}, v_{3}\right)$. Assume $r_{6} \in \gamma\left(r_{7} ; p_{3}^{\prime}, v_{2}\right)$. We have $\left(r_{4} r_{5} v_{1}\right)_{3}$, $\left(p_{1} v_{2} r_{6} r_{7} p_{3}\right)_{5}$ and a 4 -hole from the remaining points. Assume $r_{6} \in \gamma\left(r_{7} ; p_{1}, v_{2}\right)$. If $\gamma\left(r_{7} ; r_{6}^{\prime}, p_{2}\right) \neq \varnothing$, we have $\left(r_{2} r_{3} v_{3}\right)_{3},\left(p_{6} r_{7} r_{1} p_{2} v_{2}\right)_{5}$ and a 4 -hole from the remaining points where $r_{1}=\alpha\left(r_{7} ; p_{2}, r_{6}^{\prime}\right)$. If $\gamma\left(r_{7} ; r_{6}^{\prime}, p_{2}\right)=\varnothing$, we have $\left(v_{2} v_{3} p_{2}\right)_{3},\left(p_{1} r_{6} r_{7} r_{1} p_{3}\right)_{5}$ and a 4-hole from the remaining points where $r_{1}=\alpha\left(r_{7} ; p_{3}, p_{1}^{\prime}\right)$.

(b3) $\quad\left|T_{2}\right|=0$.

Let $r_{6}, r_{7} \in\left(p_{1} p_{2} p_{3}\right)$. Then we will discuss the region $\gamma\left(p_{3} ; p_{1}, v_{1}\right)$, as shown in Figure 13 .

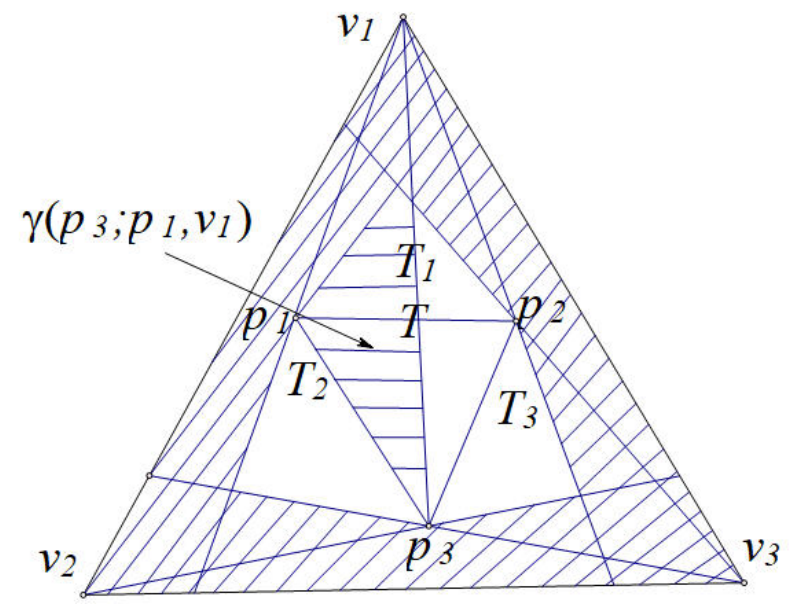

Figure 13. Figure of $\left|T_{2}\right|=0$. 
Assume $\gamma\left(p_{3} ; p_{1}, v_{1}\right)=\varnothing$. Suppose $\gamma\left(r_{6} ; p_{3}, v_{2}^{\prime}\right) \neq \varnothing$. We have $\left(p_{3} p_{1} v_{2} r_{6} \alpha\left(r_{6} ; p_{3}, v_{2}^{\prime}\right)\right)_{5}$, $\left(r_{4} r_{5} v_{1}\right)_{3}$ and a 4-hole from the remaining points. Suppose $\gamma\left(r_{6} ; p_{3}, v_{2}^{\prime}\right)=\varnothing$. If $\gamma\left(r_{7} ; r_{6}^{\prime}, p_{2}\right) \cap \gamma\left(p_{2} ; r_{7}, v_{2}^{\prime}\right) \neq \varnothing$, we have $\left(r_{2} r_{3} v_{3}\right)_{3},\left(r_{6} v_{2} p_{2} r_{1} r_{7}\right)_{5}$ and a 4-hole from the remaining points where $r_{1}=\alpha\left(r_{7} ; p_{2}, r_{6}^{\prime}\right)$. If $\gamma\left(r_{7} ; r_{6}^{\prime}, p_{2}\right) \cap \gamma\left(p_{2} ; r_{7}, v_{2}^{\prime}\right)=\varnothing$, we have $\left(v_{2} v_{3} p_{2}\right)_{3},\left(r_{1} r_{7} r_{6} p_{1} \alpha\left(r_{1} ; p_{1}, p_{3}\right)\right)_{5}$ and a 4 -hole from the remaining points where $r_{1}=\alpha\left(p_{1} ; r_{7}, p_{3}\right)$ within $\gamma\left(p_{3} ; p_{2}, v_{3}\right)$.

Assume $\gamma\left(p_{3} ; p_{2}, v_{1}\right)=\varnothing$. We have $\left(r_{2} r_{3} v_{3}\right)_{3},\left(r_{1} p_{3} r_{6} v_{2} p_{2}\right)_{5}$ and a 4-hole from the remaining points where $r_{1}=\alpha\left(p_{3} ; p_{2}, v_{3}\right)$ and $r_{6}=\alpha\left(p_{3} ; v_{2}, p_{1}\right)$.

Assume $\gamma\left(p_{3} ; p_{1}, v_{2}\right) \neq \varnothing$ and $\gamma\left(p_{3} ; p_{1}, v_{2}\right) \neq \varnothing$. We may assume $r_{6} \in \gamma\left(p_{3} ; p_{1}, v_{2}\right)$ and $r_{7} \in \gamma\left(p_{3} ; p_{1}, v_{2}\right)$. Suppose $r_{7} \in \gamma\left(r_{6} ; p_{2}, p_{1}^{\prime}\right)$. We have $\left(r_{4} r_{5} v_{1}\right)_{3},\left(r_{6} r_{7} p_{2} v_{2} p_{1}\right)_{5}$ and a 4-hole from the remaining points. Suppose $r_{7} \in \gamma\left(r_{6} ; p_{3}, p_{1}^{\prime}\right)$. If $\gamma\left(r_{7} ; r_{6}^{\prime}, p_{2}\right) \neq$ $\varnothing$, we have $\left(r_{2} r_{3} v_{3}\right)_{3},\left(r_{7} r_{6} v_{2} p_{2} r_{1}\right)_{5}$ and a 4-hole from the remaining points where $r_{1}=\alpha\left(p_{2} ; r_{7}, v_{2}^{\prime}\right)$. If $\gamma\left(r_{7} ; r_{6}^{\prime}, p_{2}\right)=\varnothing$ : and if $\gamma\left(r_{7} ; p_{1}, v_{1}\right)=\varnothing$, we have $\left(r_{2} v_{3} p_{2}\right)_{3}$, $\left(v_{1} p_{1} r_{6} r_{7} r_{4}\right)_{5}$ and a 4 -hole from the remaining points where $r_{4}=\alpha\left(r_{7} ; p_{1}, p_{3}\right)$ within $\gamma\left(p_{3} ; p_{1}, v_{1}\right)$; and if $\gamma\left(r_{7} ; p_{1}, v_{1}\right) \neq \varnothing$, we have $\left(r_{2} v_{3} p_{2}\right)_{3},\left(r_{4} p_{1} r_{6} r_{7} r_{1}\right)_{5}$ and a 4 -hole from the remaining points where $r_{4}=\alpha\left(r_{7} ; p_{1}, v_{1}\right)$.

(c) $\left|T_{1}\right|=1$. Let $r_{4} \in T_{1}$.

(c1) $\left|T_{2}\right|=1$. Let $r_{5} \in T_{2}$ and $r_{6}, r_{7} \in\left(p_{1} p_{2} p_{3}\right)$.

Firstly, consider $r_{4} \in \gamma\left(v_{1} ; p_{1}, p_{2}\right)$, then we will discuss the region $\gamma\left(v_{1} ; p_{1}, p_{2}\right) \cap$ $\left(p_{1} p_{2} p_{3}\right)=\varnothing$, as shown in Figure 14 .

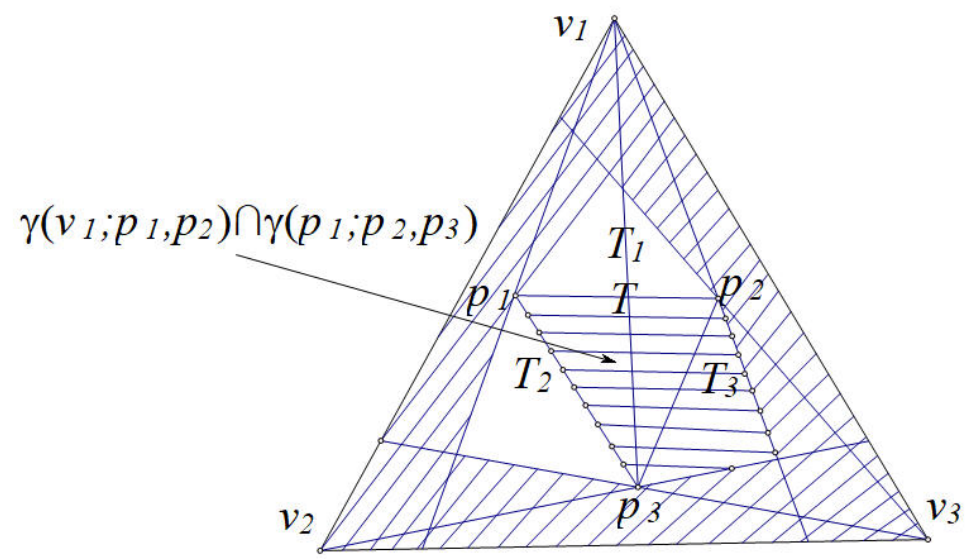

Figure 14. Figure of $\left|T_{1}\right|=1$ and $\left|T_{2}\right|=1$.

Assume $\gamma\left(v_{1} ; p_{1}, p_{2}\right) \cap\left(p_{1} p_{2} p_{3}\right)=\varnothing$. We have a cutting line $L_{5}\left(r_{4}, \alpha\left(r_{4} ; p_{2}, p_{1}^{\prime}\right)\right)$. Assume $\gamma\left(v_{1} ; p_{1}, p_{2}\right) \cap\left(p_{1} p_{2} p_{3}\right) \neq \varnothing$. Let $r_{6}=\alpha\left(p_{2} ; p_{1}, v_{1}\right)$. If $\gamma\left(r_{6} ; p_{2}, p_{1}^{\prime}\right) \neq \varnothing$, we have a cutting line $L_{5}\left(r_{4}, \alpha\left(r_{6} ; p_{2}, p_{1}^{\prime}\right)\right)$. Then we may assume $\gamma\left(r_{6} ; p_{2}, p_{1}^{\prime}\right)=\varnothing$.

Suppose $r_{5} \in \gamma\left(r_{6} ; v_{2}, r_{4}^{\prime}\right)$. If $r_{7} \in \gamma\left(r_{6} ; p_{2}^{\prime}, r_{4}\right)$, we have $\left(p_{1} v_{2} r_{5}\right)_{3}$, $\left(r_{4} r_{7} r_{6} p_{2} \alpha\left(r_{4} ; p_{2}, p_{3}\right)\right)_{5}$ and a 4-hole from the remaining points. If $r_{7} \in \gamma\left(r_{6} ; p_{1}^{\prime}, r_{4}\right)$, we have a cutting line $L_{5}\left(r_{4}, r_{6}\right)$.

Suppose $r_{5} \in \gamma\left(r_{6} ; v_{2}, p_{1}\right)$. If $\gamma\left(r_{6} ; r_{5}^{\prime}, p_{1}^{\prime}\right) \neq \varnothing$, we have $\left(v_{1} r_{4} p_{1}\right)_{3}$, $\left(r_{6} r_{5} v_{2} p_{2} \alpha\left(r_{6} ; p_{2}, r_{5}^{\prime}\right)\right)_{5}$ and a 4 -hole from the remaining points. If $\gamma\left(r_{6} ; r_{5}^{\prime}, p_{1}^{\prime}\right)=\varnothing$ : and if $r_{7} \in \gamma\left(r_{6} ; r_{4}, r_{5}^{\prime}\right)$, we have $\left(v_{2} v_{3} p_{2}\right)_{3},\left(r_{4} p_{1} r_{5} r_{6} r_{7}\right)_{5}$ and a 4-hole from the remaining points; and if $r_{7} \in \gamma\left(r_{6} ; r_{4}, p_{2}^{\prime}\right)$, we have $\left(p_{1} v_{2} r_{5}\right)_{3},\left(r_{4} r_{7} r_{6} p_{2} \alpha\left(r_{4} ; p_{2}, p_{3}\right)\right)_{5}$ and a 4 -hole from the remaining points. Suppose $r_{5} \in \gamma\left(r_{6} ; p_{2}, r_{4}^{\prime}\right)$. If $r_{7} \in \gamma\left(r_{6} ; p_{2}^{\prime}, r_{4}\right)$, 
we have $\left(p_{1} v_{2} r_{5}\right)_{3},\left(p_{2} r_{6} r_{7} r_{4} \alpha\left(r_{4} ; p_{2}, p_{3}\right)\right)_{5}$ and a 4 -hole from the remaining points. If $r_{7} \in \gamma\left(r_{6} ; r_{4}, p_{2}\right) \cap H\left(r_{6} ; r_{4} p_{2}\right)$, we have $\left(p_{1} v_{2} r_{6} r_{5}\right)_{3},\left(p_{3} r_{4} r_{7} p_{2} \alpha\left(p_{3} ; p_{2}, v_{1}^{\prime}\right)\right)_{5}$ and a 3-hole from the remaining points. If $r_{7} \in \gamma\left(p_{2} ; r_{4}, v_{1}\right)$, we have $\left(v_{1} v_{2} p_{1}\right)_{3},\left(r_{1} r_{6} r_{5} p_{2} r_{7}\right)_{5}$ and a 4 -hole from the remaining points. If $r_{7} \in \gamma\left(p_{2} ; v_{1}, p_{3}\right)$, we have $\left(p_{1} v_{2} r_{5}\right)_{3}$, $\left(v_{1} r_{4} r_{6} p_{2} r_{7}\right)_{5}$ and a 4 -hole from the remaining points.

Secondly, consider $r_{4} \in \gamma\left(v_{1} ; p_{2}, p_{3}\right)$, then we will discuss the region $\gamma\left(r_{4} ; p_{2}, p_{3}\right) \cap$ $\left(p_{1} p_{2} p_{3}\right)=\varnothing$, as shown in Figure 15

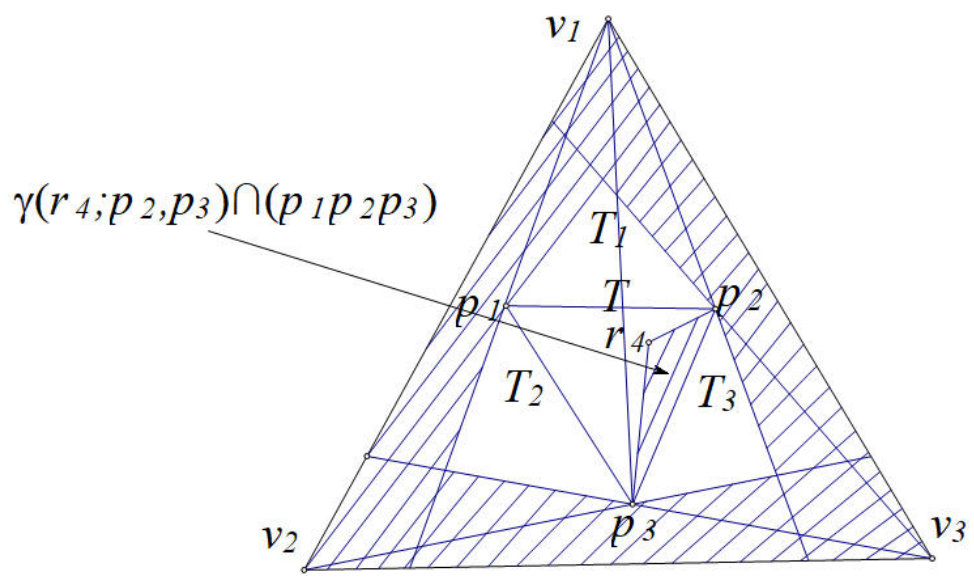

Figure 15. Figure of $\left|T_{1}\right|=1$ and $\left|T_{2}\right|=1$ with shaded region nonempty.

Assume $\gamma\left(r_{4} ; p_{2}, p_{3}\right) \cap\left(p_{1} p_{2} p_{3}\right)=\varnothing$. We have $\left(r_{2} r_{3} v_{3}\right)_{3},\left(r_{1} p_{3} r_{4} r_{6} p_{2}\right)_{5}$ and a 4-hole from the remaining points where $r_{1}=\alpha\left(p_{3} ; p_{2}, v_{3}\right), r_{6}=\alpha\left(r_{4} ; p_{2}, p_{1}\right)$. Assume $\gamma\left(r_{4} ; p_{1}, p_{2}\right) \cap\left(p_{1} p_{2} p_{3}\right)=\varnothing$. We have $L_{5}\left(p_{2}, r_{4}\right)$. Assume $\gamma\left(r_{4} ; p_{2}, p_{3}\right) \cap\left(p_{1} p_{2} p_{3}\right) \neq$ $\varnothing$ and $\gamma\left(r_{4} ; p_{1}, p_{2}\right) \cap\left(p_{1} p_{2} p_{3}\right) \neq \varnothing$. Then we may assume $r_{6} \in \gamma\left(r_{4} ; p_{2}, p_{3}\right), r_{7} \in$ $\gamma\left(r_{4} ; p_{1}, p_{2}\right)$. Suppose $r_{6} \in \gamma\left(r_{4} ; v_{1}^{\prime}, p_{3}\right) \cap\left(p_{1} p_{2} p_{3}\right)$. If $\gamma\left(r_{6} ; r_{4}^{\prime}, p_{3}\right) \neq \varnothing$, we have $\left(p_{1} r_{5} p_{2} r_{7}\right)_{4},\left(v_{1} r_{4} r_{6} r_{1} p_{3}\right)_{5}$ and $\left(r_{2} r_{3} v_{3}\right)_{3}$ where $r_{1}=\alpha\left(r_{6} ; p_{3}, r_{4}^{\prime}\right)$. If $\gamma\left(r_{6} ; r_{4}^{\prime}, p_{3}\right)=\varnothing$ : and if $r_{7} \in \gamma\left(r_{4} ; v_{1}, p_{2}\right) \cap \gamma\left(v_{1} ; p_{2}, r_{4}\right)$, we have $L_{5}\left(p_{2} ; r_{4}\right)$; and if $r_{7} \in \gamma\left(r_{4} ; r_{5}, p_{2}\right) \cap$ $\gamma\left(p_{2} ; p_{1}, v_{1}\right)$, we have $L_{5}\left(r_{4} ; r_{7}\right)$; and if $r_{7} \in \gamma\left(r_{4} ; p_{1}, r_{5}\right)$, we have $\left(v_{1} v_{2} p_{1}\right)_{3}$, $\left(r_{4} r_{7} r_{5} p_{2} r_{6}\right)_{5}$ and a 4 -hole from the remaining points. Suppose $r_{6} \in \gamma\left(r_{4} ; v_{1}^{\prime}, p_{2}\right) \cap$ $\left(p_{1} p_{2} p_{3}\right)$. If $r_{7} \in \gamma\left(v_{1} ; p_{1}, p_{2}\right) \cap\left(p_{1} p_{2} p_{3}\right)$, we have $\left(r_{5} v_{2} p_{1}\right)_{3},\left(v_{1} r_{7} p_{2} r_{6} r_{4}\right)_{5}$ and a 4-hole from the remaining points. If $r_{7} \in \gamma\left(v_{1} ; p_{2}, r_{4}\right) \cap \gamma\left(r_{4} ; p_{1}, p_{2}\right)$ : and if $r_{7} \in \gamma\left(r_{7} ; r_{4}^{\prime}, p_{1}\right)$, we have $L_{5}\left(r_{4}, r_{7}\right)$; and if $r_{5} \in \gamma\left(r_{7} ; r_{4}^{\prime}, p_{2}\right)$, we have $\left(v_{1} v_{2} p_{1}\right)_{3},\left(r_{4} r_{7} r_{5} p_{2} r_{6}\right)_{5}$ and a 4-hole from the remaining points.

(c2) $\quad\left|T_{2}\right|=0$.

Denote $r_{1}, r_{2}, r_{3} \in T_{3}, r_{4} \in T_{2}, r_{5}, r_{6}, r_{7} \in\left(p_{1} p_{2} p_{3}\right)$. Let $r_{5}=\alpha\left(p_{3} ; p_{1}, p_{2}\right)$ within $\left(p_{1} p_{2} p_{3}\right)$. If $\gamma\left(r_{5} ; p_{1}^{\prime}, p_{3}\right) \neq \varnothing$, we have $L_{5}\left(r_{5} ; \alpha\left(r_{5} ; p_{3}, p_{1}^{\prime}\right)\right)$. Then we assume $\gamma\left(r_{5} ; p_{1}^{\prime}, p_{3}\right)=\varnothing$, and we will discuss the region $\gamma\left(r_{5} ; p_{1}, p_{2}\right) \cap\left(p_{1} p_{2} p_{3}\right)=\varnothing$, as hown in Figure 16. 


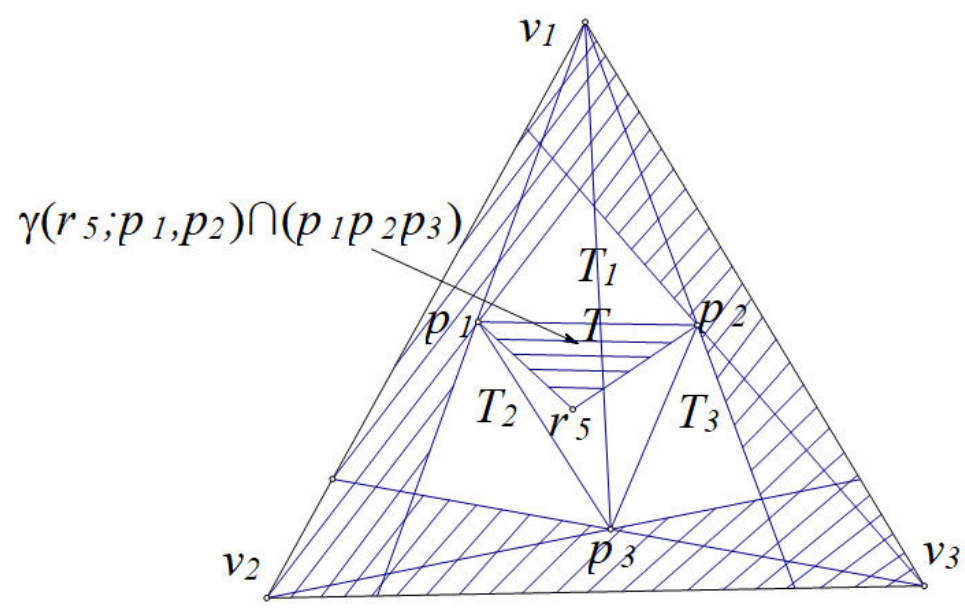

Figure 16. Figure of $\left|T_{1}\right|=1$ and $\left|T_{2}\right|=0$.

Assume $\gamma\left(r_{5} ; p_{1}, p_{2}\right) \cap\left(p_{1} p_{2} p_{3}\right)=\varnothing$, we have $\left(v_{1} r_{4} p_{3}\right)_{3},\left(r_{5} p_{1} v_{2} p_{2} \alpha\left(p_{2} ; r_{5}, p_{3}\right)\right)_{5}$ and a 4-hole from the remaining points.

Assume $\gamma\left(r_{5} ; p_{2}, p_{3}\right) \cap\left(p_{1} p_{2} p_{3}\right)=\varnothing$. Let $p_{6}=\alpha\left(r_{5} ; p_{2}, p_{3}^{\prime}\right)$. Suppose $r_{4} \in \gamma\left(r_{5} ; p_{3}, r_{6}^{\prime}\right)$. We have $\left(r_{2} r_{3} v_{3}\right)_{3},\left(p_{2} r_{1} p_{3} r_{4} \alpha\left(r_{4} ; p_{2}, p_{3}^{\prime}\right)\right)_{5}$ and a 4 -hole from the remaining points where $r_{1}=\alpha\left(p_{2} ; p_{3}, v_{2}^{\prime}\right)$. Suppose $r_{4} \in \gamma\left(r_{5} ; p_{1}, r_{6}^{\prime}\right)$. We have $\left(r_{2} r_{3} r_{4}\right)_{3},\left(p_{2} r_{1} p_{3} r_{5} r_{6}\right)_{5}$ and a 4-hole from the remaining points where $r_{1}=\alpha\left(p_{2} ; p_{3}, v_{2}^{\prime}\right)$.

Assume $\gamma\left(r_{5} ; p_{1}, p_{2}\right) \cap\left(p_{1} p_{2} p_{3}\right) \neq \varnothing$ and $\gamma\left(r_{5} ; p_{2}, p_{3}\right) \cap\left(p_{1} p_{2} p_{3}\right) \neq \varnothing$. Without loss of generality, we suppose $r_{6} \in \gamma\left(r_{5} ; p_{1}, p_{2}\right), r_{7} \in \gamma\left(r_{5} ; p_{2}, p_{3}\right)$.

Firstly, we may assume $r_{6} \in \gamma\left(r_{5} ; v_{2}, p_{2}\right)$. Suppose $r_{4} \in \gamma\left(r_{6} ; p_{7}, p_{2}^{\prime}\right)$. We have $L_{5}\left(p_{2}, r_{6}\right)$. Suppose $r_{4} \in \gamma\left(r_{5} ; r_{6}^{\prime}, p_{1}\right) \cap H\left(r_{5} ; r_{6} p_{2}\right)$. We have a cutting line $L_{5}\left(r_{5}, r_{6}\right)$. Suppose $r_{4} \in \gamma\left(r_{6} ; p_{6}^{\prime}, p_{1}^{\prime}\right)$. If $r_{7} \in \gamma\left(r_{4} ; p_{2}, p_{3}\right)$, we have $\left(v_{1} v_{2} p_{1}\right)_{3},\left(v_{4} r_{5} r_{6} p_{2} r_{7}\right)_{5}$ and a 4-hole from the remaining points. If $r_{7} \in \gamma\left(p_{2} ; r_{4}, p_{5}\right)$, we have $\left(v_{3} r_{2} r_{3}\right)_{3},\left(v_{1} p_{1} r_{6} r_{5}\right)_{4}$ and $\left(p_{3} r_{4} r_{7} p_{2} r_{1}\right)_{5}$ where $r_{1}=\alpha\left(p_{2} ; p_{3}, v_{2}^{\prime}\right)$.

Secondly, we have may assume $r_{6} \in \gamma\left(r_{5} ; v_{2}, p_{3}^{\prime}\right)$, we have $\left(v_{1} p_{1} r_{4}\right)_{3},\left(r_{5} r_{6} v_{2} p_{2} r_{7}\right)_{5}$ and a 4-hole from the remaining points.

(d) $\quad\left|T_{1}\right|=0 .\left|T_{2}\right|=0$.

Let $r_{4}, r_{5}, r_{6}, r_{7} \in\left(p_{1} p_{2} p_{3}\right)$. And $r_{1}=\alpha\left(p_{3} ; p_{2}, v_{3}\right), r_{4}=\alpha\left(p_{3} ; p_{2}, p_{1}\right), r_{5}=\alpha\left(p_{2} ; p_{1}, r_{4}\right)$. If $\gamma\left(r_{4} ; p_{2}, p_{3}^{\prime}\right) \neq \varnothing$, we have $\left(r_{2} r_{3} v_{3}\right)_{3},\left(p_{2} r_{1} p_{3} r_{4} \alpha\left(r_{4} ; p_{2}, p_{3}^{\prime}\right)\right)_{5}$ and a 4 -hole from the remaining points. Assume $r_{5} \in \gamma\left(r_{4} ; p_{1}, p_{3}\right)$. If $\gamma\left(r_{5} ; p_{2}, p_{1}^{\prime}\right) \neq \varnothing$, we have a cutting line $L_{5}\left(r_{5} ; \alpha\left(r_{5} ; p_{2}, p_{1}^{\prime}\right)\right)$. Then we will discuss the region $\gamma\left(v_{4} ; p_{1}, p_{2}^{\prime}\right) \cap\left(p_{1} p_{2} p_{3}\right)$ and $\gamma\left(r_{4} ; p_{1}, p_{3}^{\prime}\right) \cap \gamma\left(p_{1} ; p_{5}, r_{4}\right)$, as shown in Figure 17 . 


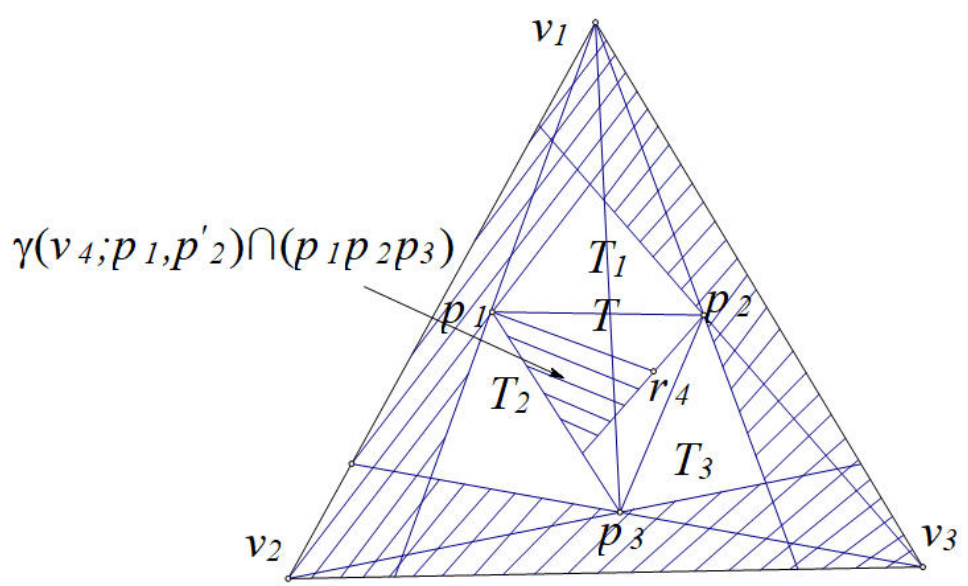

Figure 17. Figure of $\left|T_{1}\right|=1$ and $\left|T_{2}\right|=0$ with shaded region nonempty.

Assume $\gamma\left(v_{4} ; p_{1}, p_{2}^{\prime}\right) \cap\left(p_{1} p_{2} p_{3}\right)=\varnothing$. We have $\left(r_{7} r_{5} v_{2}\right)_{3},\left(r_{4} p_{3} v_{1} p_{1} r_{6}\right)_{5}$ and a 4-hole from the remaining points where $r_{6}=\alpha\left(r_{4} ; p_{1}, p_{3}^{\prime}\right)$.

Assume $\gamma\left(r_{4} ; p_{1}, p_{3}^{\prime}\right) \cap \gamma\left(p_{1} ; p_{5}, r_{4}\right)=\varnothing$. Let $r_{6}=\alpha\left(p_{1} ; p_{3}, r_{4}\right)$. Suppose $r_{7} \in \gamma\left(r_{6} ; r_{4}, p_{1}^{\prime}\right)$. We have $\left(v_{1} v_{2} p_{1}\right)_{3},\left(p_{2} r_{4} r_{7} r_{6} r_{5}\right)_{5}$ and a 4 -hole from the remaining points. Suppose $r_{7} \in \gamma\left(r_{6} ; r_{4}, v_{1}^{\prime}\right) \cap \gamma\left(r_{4} ; p_{1}, p_{2}^{\prime}\right)$. We have $\left(r_{1} r_{2} r_{3}\right)_{3},\left(p_{1} v_{2} p_{2} r_{5}\right)_{4}$ and $\left(r_{4} p_{3} v_{1} r_{6} r_{7}\right)_{5}$. Suppose $r_{7} \in \gamma\left(r_{6} ; r_{5}, v_{1}^{\prime}\right) \cap \gamma\left(r_{4} ; p_{1}, p_{2}^{\prime}\right)$. We have $\left(v_{3} r_{2} r_{3}\right)_{3},\left(r_{4} p_{2} r_{1} p_{3}\right)_{4}$ and $\left(r_{6} v_{1} p_{2} r_{5} r_{7}\right)_{5}$. Suppose $r_{7} \in \gamma\left(r_{6} ; r_{5}, p_{2}^{\prime}\right)$. We have $\left(v_{1} v_{2} p_{2}\right)_{3},\left(r_{4} p_{3} r_{6} r_{7} r_{5}\right)_{5}$ and a 4 -hole from the remaining points. Suppose $r_{7} \in \gamma\left(r_{6} ; p_{1}, r_{3}^{\prime}\right)$. We have a cutting line $L_{5}\left(p_{3}, r_{6}\right)$.

Assume $\gamma\left(v_{4} ; p_{1}, p_{2}^{\prime}\right) \cap\left(p_{1} p_{2} p_{3}\right) \neq \varnothing$ and $\gamma\left(r_{4} ; p_{1}, p_{3}^{\prime}\right) \cap \gamma\left(p_{1} ; p_{5}, r_{4}\right) \neq \varnothing$. Without loss of generality, assume $r_{6} \in \gamma\left(r_{4} ; p_{1}, p_{2}^{\prime}\right) \cap\left(p_{1} p_{2} p_{3}\right), r_{7} \in \gamma\left(r_{4} ; p_{1}, p_{3}^{\prime}\right) \cap \gamma\left(p_{1} ; p_{5}, r_{4}^{\prime}\right)$.

Suppose $r_{6} \in \gamma\left(r_{5} ; p_{3}, p_{1}^{\prime}\right)$. We have a cutting line $L_{5}\left(r_{6}, \alpha\left(r_{6} ; p_{1}, p_{3}^{\prime}\right)\right.$.

Suppose $r_{6} \in \gamma\left(r_{5} ; p_{3}, p_{1}\right) \cap \gamma\left(v_{1} ; r_{4}, p_{3}\right)$. If $r_{7} \in \gamma\left(r_{5} ; p_{3}, p_{2}^{\prime}\right) \cap \gamma\left(p_{1} ; r_{5}, r_{4}\right)$, we have $\left(v_{2} p_{2} r_{5}\right)_{3}$, $\left(v_{1} p_{1} r_{7} r_{4} r_{6}\right)_{5}$ and a 4 -hole from the remaining points. If $r_{7} \in \gamma\left(r_{5} ; p_{3}^{\prime}, p_{1}^{\prime}\right) \cap \gamma\left(r_{4} ; p_{1}, p_{3}^{\prime}\right)$, we have $\left(v_{2} p_{2} p_{3}\right)_{3},\left(v_{1} p_{1} r_{5} r_{7} r_{6}\right)_{5}$ and a 4 -hole from the remaining points.

Suppose $r_{6} \in \gamma\left(r_{5} ; p_{3}, p_{1}\right) \cap \gamma\left(v_{1} ; r_{1}, p_{1}\right)$. If $r_{7} \in \gamma\left(r_{6} ; r_{4}, v_{1}^{\prime}\right)$, we have $\left(v_{1} r_{6} r_{7} r_{4} p_{3}\right)_{5}$, $\left(p_{1} v_{2} p_{2} r_{5}\right)_{4}$ and $\left(r_{1} r_{2} r_{3}\right)_{3}$. If $r_{7} \in \gamma\left(r_{6} ; r_{5}, v_{1}^{\prime}\right)$, we have $\left(v_{2} v_{3} p_{2}\right)_{3},\left(v_{1} p_{1} r_{5} r_{7} r_{6}\right)_{5}$ and a 4-hole from the remaining points.

(6) $\left|T_{3}\right|=2$.

Let $r_{1}, r_{2} \in T_{3}$ and $r_{1}=\alpha\left(p_{2} ; p_{3}, v_{1}^{\prime}\right)$. Assume $r_{2} \in \gamma\left(r_{1} ; p_{2}, v_{3}\right)$. We have $\left(p_{2} r_{1} r_{2} v_{3}\right)_{4}$ and the remaining 9 points are in $\boldsymbol{H}\left(\overline{v_{3}} ; p_{2} p_{3}\right)$, as shown in Figure 18. 


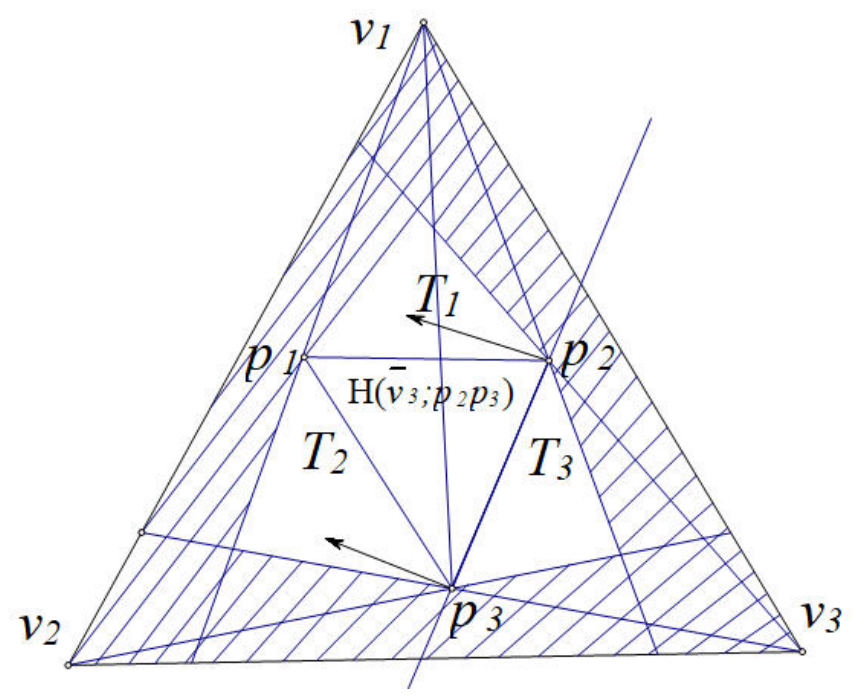

Figure 18. Figure of $\left|T_{3}\right|=2$.

By the discussion of Part One, we know our conclusion is right. Assume $r_{2} \in \gamma\left(r_{1} ; p_{3}^{\prime}, v_{2}\right)$. We have $\left(p_{3} r_{1} r_{2} v_{2}\right)_{4}$. By the discussion of Part One, we know our conclusion is also right. Assume $r_{2} \in \gamma\left(r_{1} ; p_{3}^{\prime}, p_{2}\right)$. We have a cutting line $L_{5}\left(p_{2}, \alpha\left(p_{2} ; p_{3}, p_{1}\right)\right)$.

(7) $\left|T_{3}\right|=1$.

Let $r_{1} \in T_{3}, r_{2} \in T_{1}, r_{3} \in T_{2}$ and $r_{4}, r_{5}, r_{6}, r_{7} \in\left(p_{1} p_{2} p_{3}\right)$. Let $r_{4}=\alpha\left(p_{3} ; p_{2}, p_{1}\right)$ within $\left(p_{1} p_{2} p_{3}\right)$. Assume $r_{4} \in \gamma\left(p_{3} ; p_{1}, v_{1}\right)$, as shown in Figure 19 .

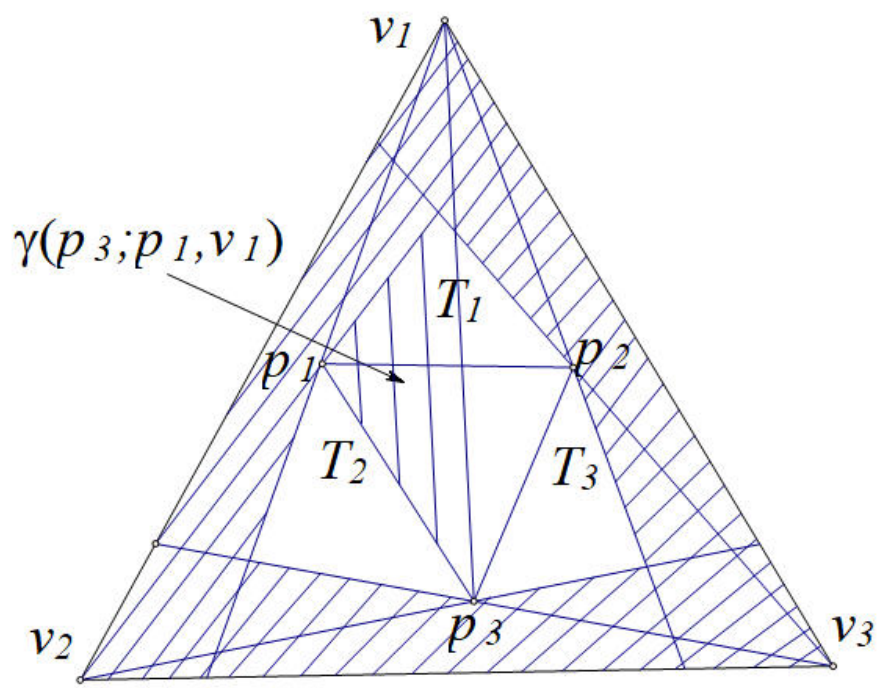

Figure 19. Figure of $\left|T_{3}\right|=1$.

If $r_{2} \in \gamma\left(v_{1} ; p_{2}, p_{3}\right)$, we have a cutting line $L_{5}\left(r_{2}, \alpha\left(r_{2} ; p_{2}, p_{1}\right)\right)$. If $r_{2} \in \gamma\left(v_{1} ; p_{2}, p_{1}\right)$, we have a cutting line $L_{5}\left(v_{1}, \alpha\left(v_{1} ; p_{2}, p_{1}\right)\right)$. Assume $r_{4} \in \gamma\left(p_{3} ; p_{2}, v_{1}\right)$. If $\gamma\left(r_{4} ; p_{3}, p_{2}^{\prime}\right) \neq \varnothing$, we have a cutting line $L_{5}\left(r_{4}, \alpha\left(r_{4} ; p_{3}, p_{2}^{\prime}\right)\right)$. If $\gamma\left(r_{4} ; p_{2}, p_{3}^{\prime}\right) \neq \varnothing$, we have a cutting line $L_{5}\left(r_{4} ; \alpha\left(r_{4} ; p_{2}, p_{3}^{\prime}\right)\right)$. If $\gamma\left(r_{4} ; p_{3}, p_{2}^{\prime}\right)=\varnothing$ and $\gamma\left(r_{4} ; p_{2}, p_{3}^{\prime}\right)=\varnothing$ : and if $r_{1} \in \gamma\left(r_{4} ; p_{2}, v_{3}\right)$, we have $\left(r_{4} p_{3} v_{3} r_{1}\right)_{4} ;$ and if $r_{1} \in \gamma\left(r_{4} ; p_{3}, v_{3}\right)$, we have $\left(p_{2} r_{4} r_{1} v_{3}\right)_{4}$. Then the remaining 9 points are all in $H\left(\overline{v_{3}} ; p_{2} p_{3}\right)$. By the discussion of Part One, our conclusion is right.

(8) $\left|T_{3}\right|=0$. 
Then $\left|T_{2}\right|=0,\left|T_{1}\right|=0$ and $r_{i} \in\left(p_{1} p_{2} p_{3}\right)$ for $i=1, \ldots, 7$. Let $r_{1}=\alpha\left(p_{1} ; p_{3}, p_{2}\right)$.

If $r_{1} \in \gamma\left(p_{1} ; p_{3}, v_{3}\right)$, as shown in Figure 20 .

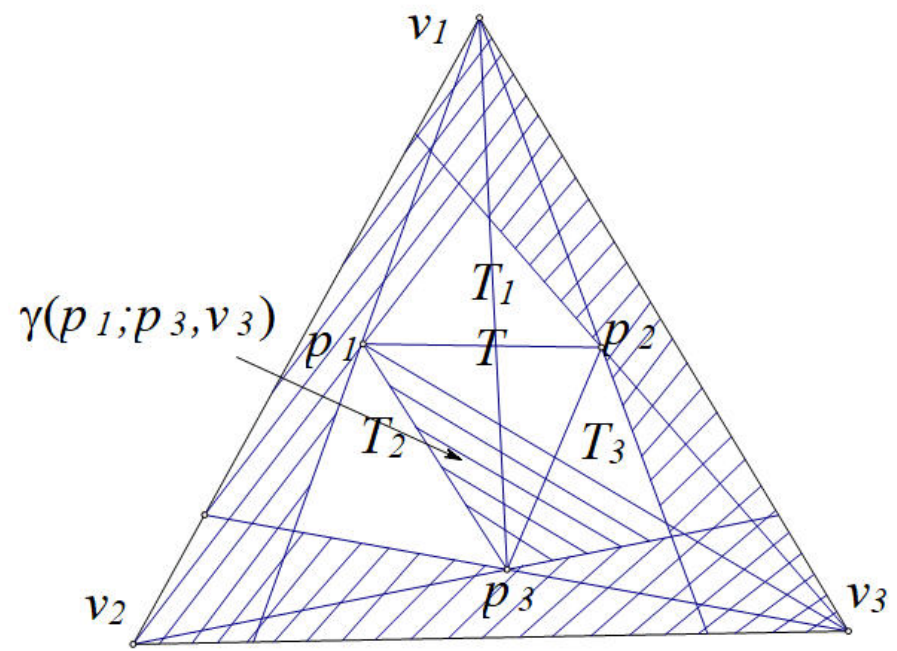

Figure 20. Figure of $\left|T_{1}\right|=0$ and $\left|T_{2}\right|=0$.

We have $\left(v_{1} p_{1} r_{1} p_{3}\right)_{4}$ and the remaining 9 points are all in $H\left(\overline{p_{3}} ; p_{1} r_{1}\right)$. By the discussion of Part One, our conclusion is right. If $r_{1} \in \gamma\left(p_{1} ; p_{3}, v_{3}\right)$ : and if $\gamma\left(r_{1} ; p_{1}, p_{3}^{\prime}\right)=\varnothing$, we have $\left(v_{1} p_{3} r_{1} p_{1}\right)_{4}$ and the remaining 9 points are all in $H\left(\bar{v}_{1} ; p_{3} r_{1}\right)$; and if $\gamma\left(r_{1} ; p_{1}, p_{3}^{\prime}\right) \neq \varnothing$, we have a cutting line $L_{5}\left(r_{1}, \alpha\left(r_{1} ; p_{1}, p_{3}^{\prime}\right)\right)$.

\section{Conclusions}

In this paper, we discuss a classical discrete geometry problem. After detailed proof, conclusion shows that a general planar point set contains a 3-hole, a 4-hole and a 5-hole, with at least 13 points. As $30 \leq n(6) \leq 463[16,21]$ and $n(7)$ does not exist, the proposed theorem will contribute to the theoretical research to some degree. Discrete geometry is a meaningful tool to study social networks. Therefore, our conclusion could be used to deal with some complex network problems. For example, under the environment of competition social structure, the structural holes which have been studied by many economists, are part of an important research branch of discrete geometry.

Author Contributions: Conceptualization, Z.Y.; Funding acquisition, Q.Y.; Methodology, Q.Y. and X.Y.

Funding: National Social Science Fund of China (18CGL018).

Acknowledgments: National Social Science Fund of China (18CGL018). This fund covers the costs to publish in open access.

Conflicts of Interest: The author declares no conflict of interest.

\section{References}

1. Erdős, P.; Szekers, G. A combinatorial problem in geometry. Compos. Math. 1935, 2, 463-470.

2. Erdős, P.; Szekers, G. On some extremum problem in elementary geometry. Ann. Univ. Sci. Budapest 1960, 3, 53-62.

3. Szekeres, G.; Peters, L. Computer solution to the 17-point Erdős-Szekeres problem. ANZIAM J. 2006, 48, 151-164. [CrossRef]

4. Harborth, H. Konvexe Funfeck in ebenen Punktmengen. Elem. Math. 1978, 33, 116-118.

5. Horton, J. Sets with no empty convex 7-gons. Can. Math. Bull. 1983, 26, 482-484. [CrossRef]

6. Gerken, T. Empty convex hexagons in planar point sets. Discret. Comput. Geom. 2008, 39, 239-272. [CrossRef]

7. Nicolás, C. The empty hexagon theorem. Discret. Comput. Geom. 2007, 38, 389-397. [CrossRef]

8. Urabe, M. On a partition into convex polygons. Discret. Appl. Math. 1996, 64, 179-191. [CrossRef] 
9. Hosono, K.; Urabe, M. A minimal planar point set with specified disjoint empty convex subsets. Lect. Notes Comput. Sci. 2008, 4535, 90-100.

10. Hosono, K.; Urabe, M. On the number of disjoint convex quadrilaterals for a planar point set. Comput. Geom. Theory Appl. 2001, 20, 97-104. [CrossRef]

11. Hosono, K.; Urabe, M. On the minimum size of a point set containing two non-intersecting empty convex polygons. Lect. Notes Comput. Sci. 2005, 3742, 117-122.

12. Wu, L.; Ding, R. Reconfirmation of two results on disjoint empty convex polygons. Lect. Notes Comput. Sci. 2007, 4381, 216-220.

13. Bhattacharya, B.; Das, S. On the minimum size of a point set containing a 5-hole and a disjoint 4-hole. Stud. Sci. Math. Hung. 2011, 48, 445-457. [CrossRef]

14. Bhattacharya, B.B. Sandip Das, On pseudo-convex partitions of a planar point set. Discret. Math. 2013, 313, 2401-2408. [CrossRef]

15. Bhattacharya, B.; Das, S. Disjoint Empty Convex Pentagons in Planar Point Sets. Period. Math. Hung. 2013, 66, 73-86. [CrossRef]

16. Hosono, K.; Urabe, M. Specifed holes with pairwise disjoint interiors in planar point sets. AKCE Int. J. Graph. Comb. 2018. [CrossRef]

17. You, X.; Wei, X. On the minimum size of a point set containing a 5-hole and double disjoint 3-holes. Math. Notes 2013, 97, 951-960. [CrossRef]

18. You, X.; Wei, X. A note on the upper bound for disjoint convex partitions. Math. Notes 2014, 96, $268-274$. [CrossRef]

19. You, X.; Wei, X. A note on the value about a disjoint convex partition problem. Ars Comb. 2014, 115, 459-465.

20. You, X.; Chen, T. A note on the value about a disjoint convex partition problem. Math. Notes, 2018, 104, $135-149$.

21. Koshelev, V.A. On Erdős-Szekeres problem for empty hexagons in the plane. Modelirovanie i Analiz Informatsionnykh Sistem 2009, 16, 22-74.

(C) 2018 by the authors. Licensee MDPI, Basel, Switzerland. This article is an open access article distributed under the terms and conditions of the Creative Commons Attribution (CC BY) license (http:/ / creativecommons.org/licenses/by/4.0/). 